QUARTERLY OF APPLIED MATHEMATICS

VOLUME LXIV, NUMBER 2

JUNE 2006, PAGES 201-228

S $0033-569 \times(06) 00970-7$

Article electronically published on May 3, 2006

\title{
TRANSIENT TEMPERATURE AND ELASTIC RESPONSE OF A SPACE-BASED MIRROR IN THE RADIATION-CONDUCTION ENVIRONMENT
}

\author{
BY \\ BEJOY K. CHOUDHURY \\ Lockheed Martin Space Systems, Sunnyvale, California 94089
}

\begin{abstract}
The problem of the one-dimensional heat equation with radiation boundary conditions is studied. Steady state and transient solutions for a space-based mirror are obtained under a broad class of boundary specification. Associated elastic stress and displacement are investigated. Time-varying heat flux at the boundary is also considered. The method requires solving equations of the type $\tan \lambda=\left(B i_{1}+B i_{2}\right) \lambda /\left(\lambda^{2}-B i_{1} B i_{2}\right)$ for the eigenvalues $\lambda$, for which complete asymptotic solutions are given. Selected transient response results for temperature and stresses are presented.
\end{abstract}

1. Introduction. We consider a space-based mirror that exchanges heat by radiation to space on one side and to a housing structure on the other side. The mirror is idealized to be a flat plate of uniform thickness, the plate thickness being small compared to the plate's width. The boundary temperatures at each end are unequal. In addition, heat flux at the boundary is also specified. The heat flux is constant when the mirror is stationary, but otherwise the flux may vary with time during the mirror's movement. The space is assumed to be at the $5^{\circ} \mathrm{K}$ effective temperature. Thermophysical, transport, and elastic properties of the plate material are assumed to be homogeneous, isotropic, and constant for the range of temperatures of our interest. Temperature variation in the transverse directions is small compared to the variation through the plate's thickness. There is no heat generation. Some assumptions, especially the last two, are not necessary in the formulation but are assumed merely to keep the algebra to the minimum.

We intend to study temperature and stress-displacement in the mirror by solving Fourier's heat conduction equation and a set of uncoupled elastic equations under a general class of boundary conditions. An associated vibration problem due to the sudden heating or cooling was not investigated. Exact solutions in closed-form with radiation boundary conditions are rare. Lacking a general solution for the eigenvalues, the practice

Received December 10, 2004.

2000 Mathematics Subject Classification. Primary 74B05, 74F05, $42 \mathrm{~B} 05$.

Key words and phrases. Heat conduction, thermoelasticity, Fourier series.

E-mail address: bejoy.choudhury@lmco.com

(C)2006 Brown University

Reverts to public domain 28 years from publication 
has been to present transient responses in graphical charts [14, [22, with occasional tabulation of the first few Fourier constants [8], [18. However, the charts cannot be read with good accuracy, and due to the generally slow convergence of the series it becomes necessary to take a large number of terms. We show here how transient solutions may be obtained easily and accurately for a variety of conditions. Interest in the closed-form solutions continues because they are simple to use and because accuracy and stability of solutions may be studied by classical analysis. The analytical solution can reveal some fine structure inherent in the problem and may itself suggest further generalization.

Primary references for this work are [8] for conduction heat transfer, and [6] for thermoelasticity. Both sources provide very thorough and authoritative treatment of the material. Standard references for Fourier series are [13, [25], 28]. Additional references are 21], 23], 19].

2. Formulation of the problem. We seek a solution to the one-dimensional heat equation with the following set of boundary conditions:

$$
\left.\begin{array}{rlrl}
\rho c \frac{\partial T}{\partial t} & =\kappa \frac{\partial^{2} T}{\partial z^{2}}, & & 0 \leq \leq L, \quad t \geq 0, \\
T & =T_{0}, & t & =0, \quad 0 \leq z \leq L, \\
\kappa \frac{\partial T}{\partial z} & =\sigma \epsilon_{1} \mathcal{F}_{1}\left(T^{4}-T_{1}^{4}\right), & & z=0, \quad t>0, \\
-\kappa \frac{\partial T}{\partial z} & =\sigma \epsilon_{2} \mathcal{F}_{2}\left(T^{4}-T_{2}^{4}\right), & & z=L, \quad t>0,
\end{array}\right\}
$$

where $\epsilon_{1}, \mathcal{F}_{1}$ are the emissivity and radiation view factors for the side $z=0, \epsilon_{2}, \mathcal{F}_{2}$ are the same for the side $z=L$, and $\sigma$ is a Stefan-Boltzmann constant. See Fig. 1. The initial temperature $T_{0}$ may be a function of $z$. Variables $\rho, c, \kappa$ are the density, specific heat, and thermal conductivity of the mirror material. The right-hand sides of $(2.1 \mathrm{c}-\mathrm{d})$ will be linearized as $h_{i}\left(T-T_{i}\right)$, where $h_{i}$ is heat transfer coefficient for the surface $i$. In particular,

$$
\begin{aligned}
& h_{1}=4 \sigma \epsilon_{1} \mathcal{F}_{1} T_{1}^{3}+O\left(\frac{T-T_{1}}{T_{1}}\right), \\
& h_{2}=\sigma \epsilon_{2} \mathcal{F}_{2}\left(T_{1}+T_{2}\right)\left(T_{1}^{2}+T_{2}^{2}\right)+O\left(\frac{T-T_{1}}{T_{1}+T_{2}}\right) .
\end{aligned}
$$

Note that $h_{1}$ and $h_{2}$ are linearized differently. This is because in the first case, temperature difference $\Delta T$ between the inner face at $z=0$ and the boundary temperature $T_{1}$ is small. Hence $\left|\left(T-T_{1}\right) / T_{1}\right| \ll 1$. In the second case, the outer face at $z=L$ is exposed to space at $T_{2} \approx 5^{\circ} \mathrm{K}$, but the mirror will be kept near room temperature $\left(\approx 293^{\circ} \mathrm{K}\right)$. Therefore, $\left|\left(T-T_{2}\right) / T_{2}\right| \gg 1$, and the same approximation cannot be used. The dimensionless term $\sigma \epsilon \mathcal{F} T^{3} L / \kappa$ has been called the Stark number.

Appropriate dimensionless parameters are the Biot numbers, $B i_{i}=h_{i} L / \kappa, i=1$ or 2 , and the Fourier number $F_{O}=s t / L^{2}$, where $s=\kappa /(\rho c)$ is thermal diffusivity of the material. We now transform boundary conditions $(2.1 \mathrm{c}-\mathrm{d})$ into homogeneous conditions of the type $\alpha_{1} \varphi(0)+\beta_{1} \varphi^{\prime}(0)=0, \alpha_{2} \varphi(L)+\beta_{2} \varphi^{\prime}(L)=0$ where $\alpha_{1}, \beta_{1}, \alpha_{2}, \beta_{2}$ are 


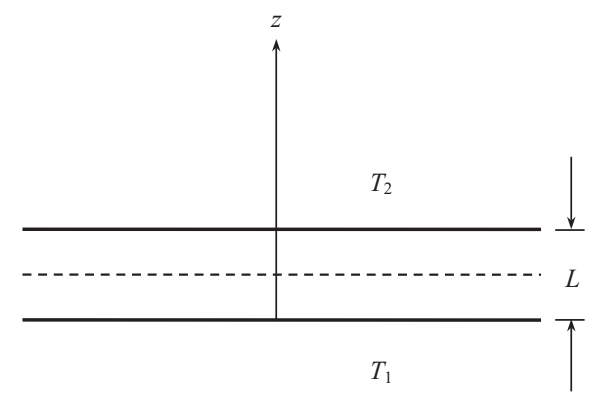

FIG. 1. Infinite plate with radiation boundary conditions

constants. We use the linear transformation $T=a_{0}+b_{0} z+\varphi$ [25], p. 305, and choose

$$
a_{0}=T_{1}+\frac{B i_{2}\left(T_{2}-T_{1}\right)}{B i_{1}+B i_{2}+B i_{1} B i_{2}}, \quad b_{0} L=\frac{B i_{1} B i_{2}\left(T_{2}-T_{1}\right)}{B i_{1}+B i_{2}+B i_{1} B i_{2}} .
$$

The transformed equations will not be shown here. The equations form a regular SturmLiouville system with an infinite sequence of real positive eigenvalues $\lambda_{0}<\lambda_{1}<\lambda_{2}<\cdots$, and

$$
\lim _{n \rightarrow \infty} \lambda_{n}=+\infty
$$

ultimately. For separated endpoint conditions and assuming $\beta_{1} \beta_{2} \neq 0$, it is known that as $n \rightarrow \infty$, the eigenvalues satisfy the asymptotic relation

$$
\lambda_{n}=n \pi+O\left(\frac{1}{n}\right) .
$$

A similar result exists for the eigenfunctions [4]. We shall define the order term more precisely later. It should be mentioned that the separation property of the eigenvalues may not be used to determine sequentially the roots, as the process may be oscillatory or unstable.

3. The temperature solution. Let $\lambda_{n}, \chi_{n}$ be the eigenvalue and eigenfunction of the transformed Sturm-Liouville system and let $z^{\prime}=z / L$. We construct the eigenfunction $\chi_{n}$ as

$$
\chi_{n}=\sin \left(\lambda_{n} z^{\prime}\right)+\frac{\lambda_{n}}{B i_{1}} \cos \left(\lambda_{n} z^{\prime}\right) .
$$

The eigenfunctions are orthogonal and have the properties,

$$
\int_{0}^{1} \chi_{m} \chi_{n} d z^{\prime}= \begin{cases}0 & (m \neq n), \\ N\left(\lambda_{n}\right) & (m=n),\end{cases}
$$

where

$$
N\left(\lambda_{n}\right)=\frac{1}{2 B i_{1}^{2}} \cdot \frac{\left(\lambda_{n}^{2}+B i_{1}^{2}\right)\left(\lambda_{n}^{2}+B i_{2}^{2}\right)+\left(B i_{1}+B i_{2}\right)\left(\lambda_{n}^{2}+B i_{1} B i_{2}\right)}{\lambda_{n}^{2}+B i_{2}^{2}} .
$$


Equation (3.3) is quite general and has been known for some time, e.g., see [21. The solution to $((2.1 \mathrm{a}-\mathrm{d}))$ can be written as

$$
\left.\begin{array}{rl}
\theta & =\frac{T-T_{1}}{T_{2}-T_{1}}=c\left(1+B i_{1} z^{\prime}\right)+\sum_{n=0}^{\infty} A_{n} \chi_{n} \exp \left(-\lambda_{n}^{2} s t / L^{2}\right)\left(T_{1} \neq T_{2}\right), \\
A_{n} & =\left[N\left(\lambda_{n}\right)\right]^{-1} \int_{0}^{1}\left[\frac{T_{0}-T_{1}}{T_{2}-T_{1}}-c\left(1+B i_{1} z^{\prime}\right)\right] \chi_{n} d z^{\prime}, \\
c & =\frac{B i_{2}}{B i_{1}+B i_{2}+B i_{1} B i_{2}},
\end{array}\right\}
$$

where $\lambda_{n}$ are nonnegative roots of the transcendental equation

$$
\lambda_{n} \cot \lambda_{n}=\frac{\lambda_{n}^{2}-B i_{1} B i_{2}}{B i_{1}+B i_{2}} .
$$

The eigenvalues of a regular Sturm-Liouville system are real. The substitution of $\lambda_{n}=-\lambda_{n}$ leaves (3.5) and the product $A_{n} \chi_{n}$ in (3.4a) unchanged. Thus, it is sufficient to consider only the positive roots as the negative roots contain no new information.

The following integrals will be needed later:

$$
\begin{aligned}
J_{n} & =\int_{0}^{1} \chi_{n} d z^{\prime}=\frac{1}{\lambda_{n}}\left(1-\cos \lambda_{n}+\frac{\lambda_{n}}{B i_{1}} \sin \lambda_{n}\right), \\
K_{n} & =\int_{0}^{1} \chi_{n} z^{\prime} d z^{\prime}=\frac{1}{\lambda_{n} B i_{1}}\left[\frac{1}{\lambda_{n}}\left(\lambda_{n}^{2}+B i_{1}\right) \sin \lambda_{n}+\left(1-B i_{1}\right) \cos \lambda_{n}-1\right] .
\end{aligned}
$$

If $B i_{1}=0$ (the end at $z=0$ insulated), then $\lim _{B i_{1} \rightarrow 0} B i_{1} \chi_{n}=\lambda_{n} \cos \left(\lambda_{n} z^{\prime}\right), c=1$, and the temperature solution becomes

$$
\left.\begin{array}{l}
\frac{T}{T_{2}}=1+\sum_{n=0}^{\infty} A_{n} \cos \left(\lambda_{n} z^{\prime}\right) \exp \left(-\lambda_{n}^{2} s t / L^{2}\right) \quad\left(T_{2} \neq 0\right), \\
A_{n}=2 \frac{\lambda_{n}^{2}+B i_{2}^{2}}{\lambda_{n}^{2}+B i_{2}^{2}+B i_{2}} \int_{0}^{1}\left(\frac{T_{0}}{T_{2}}-1\right) \cos \left(\lambda_{n} z^{\prime}\right) d z^{\prime} .
\end{array}\right\}
$$

The eigenvalues are nonnegative roots of $\lambda_{n} \tan \lambda_{n}=B i_{2}$.

If $B i_{1}=B i_{2}=0$, the Sturm-Liouville problem reduces to a homogeneous system with Neumann conditions. The eigenvalues are $\lambda_{n}=n \pi, n=0,1,2, \ldots$. Here the trivial root $\lambda_{0}=0$ is also an eigenvalue.

If the boundary temperatures are equal but the heat transfer rates are different $\left(T_{1}=T_{2}, B i_{1} \neq B i_{2}\right)$, then the solution for a constant $T_{0}$ is

$$
\frac{T-T_{1}}{T_{0}-T_{1}}=\sum_{n=0}^{\infty}\left[N\left(\lambda_{n}\right)\right]^{-1}\left(1-\cos \lambda_{n}+\frac{\lambda_{n}}{B i_{1}} \sin \lambda_{n}\right) \frac{\chi_{n}}{\lambda_{n}} \exp \left(-\lambda_{n}^{2} s t / L^{2}\right) .
$$

Note that $T_{0} \neq T_{1}$, for then the temperature will be unchanged.

Equations of the type (3.5) appear frequently in vibration, and in heat conduction problems in plane and spherical geometries. The equation also appears in the Green's 
function formulation. Fourier realized the importance of the roots as in his seminal treatise, Théorie Analytique de la Chaleur (1822), he devotes considerable space to the determination. Tables of roots for the simpler cases have been published [8, [1], 18, but a general solution has not been reported. The solution is believed to be difficult and it has been remarked that the roots cannot be found by ordinary algebraic methods (S. Kakaç and Y. Yener, Heat Conduction, 3rd ed., Taylor and Francis, London, p. 141, 1993).

We intend to show here that an elementary solution exists. Indeed, we present the complete solution obtained by two methods. The first method uses basic ideas of asymptotic expansion. The second method uses elementary trigonometrical properties. When either of the solutions is combined with Newton-Raphson or other iterative schemes, the roots will be calculated to any desired accuracy.

4. Uniqueness, convergence, and summability. The theory of Fourier series is rich and extensive. Pivotal to the construction of the series are the following questions: a) validity of the term-by-term integration, b) uniqueness of the solution, c) uniqueness of the Fourier constants, d) convergence and summability of the series. It is not the intention to review here many delicate points as we are mainly concerned in the application to physical problems.

A Fourier series may be integrated term-by-term after multiplication by any function of bounded variation [13, p. 95, 24]. Uniqueness of the solution can be proved by considering the integral

$$
J(t)=\iiint \frac{1}{2} v^{2} d \Sigma,
$$

the integral being taken over the entire body. Here $v=T_{1}-T_{2}$, and $T_{1}, T_{2}$ are two possible solutions. If $T$ is continuous, it can be shown that the integral must vanish, proving the uniqueness of the solution.

The uniqueness of the Fourier trigonometrical coefficients is proved in a theorem due to Riemann, du Bois-Reymond, and Cantor [24], p. 428. For Perseval's and Riesz-Fisher theorems see [12], pp. 20, 348.

The Riemann-Lebesgue theorem shows that Fourier coefficients of any integrable function tend to zero [13], p. 23, [12], p. 19. Hardy, extending Fatou's result, has shown that

$$
a_{n}=O\left(\frac{1}{n}\right), \quad b_{n}=O\left(\frac{1}{n}\right)
$$

$a_{n}, b_{n}$ being the two Fourier coefficients. For convergence and summability of the Fourier series see [12, 13], 28]. The summability question is more important than the convergence because it is known that there exist continuous functions whose Fourier series are divergent at some points.

In this paper we shall assume that the functions to be represented by Fourier series satisfy Dirichlet conditions, are of the bounded variation, and Lebesgue integrable. Although no specific reference has been made, the space is $L_{2}$, a Hilbert space. 
5. Eigenvalues: the asymptotic solution. Roots of the transcendental equation

$$
\tan \lambda=\frac{\left(B i_{1}+B i_{2}\right) \lambda}{\lambda^{2}-B i_{1} B i_{2}}, \quad B i_{1} \geq 0, B i_{2} \geq 0,
$$

are determined by the intersections between the curves $y=\tan \lambda$ and $y=\left(B i_{1}+B i_{2}\right) \lambda$ $/\left(\lambda^{2}-B i_{1} B i_{2}\right)$. The number of such intersections is infinite and therefore (5.1) has an infinite number of roots. The right-hand side of (5.1) has two $y$-asymptotes at $\lambda=$ $\pm \sqrt{ }\left(B i_{1} B i_{2}\right)$; see Fig. 2. Note that the equation is trivially satisfied by $\lambda=0$. The asymptotic method used here is described in [20, [11. In the derivations we have used some inequality properties of fractions [17.

Lemma. Let $a, b$ be the sum and product of two Biot numbers $B i_{1}, B i_{2}$, and let $\mu=$ $\frac{1}{2}\left[a+\sqrt{ }\left(a^{2}+4 b\right)\right]$. Then for real nonnegative values of $\lambda,\left|a \lambda /\left(\lambda^{2}-b\right)\right|<1$ for $\lambda>\mu$, and $\left|a \lambda /\left(\lambda^{2}-b\right)\right|>1$ for $\lambda<\mu$.

Proof. We have $a=B i_{1}+B i_{2}, b=B i_{1} B i_{2}$. Since Biot numbers are real and positive, $a \geq 0, b \geq 0, a^{2}-4 b \geq 0, \mu \geq 0$. Therefore, $\left|a \lambda /\left(\lambda^{2}-b\right)\right|<1$ means

$$
\lambda^{4}-\left(a^{2}+2 b\right) \lambda^{2}+b^{2}>0 .
$$

Factorizing the left-hand side it is seen that $\left|a \lambda /\left(\lambda^{2}-b\right)\right|<1$ when $\lambda^{2}>\mu^{2}$, or $\lambda>\mu$. Similarly, $\left|a \lambda /\left(\lambda^{2}-b\right)\right|>1$ when $\lambda^{2}<\mu^{2}$, or $\lambda<\mu$.

With the help of the lemma we can write the nonnegative roots of (5.1) as

$$
\lambda= \begin{cases}n \pi+\tan ^{-1}\left(\frac{a \lambda}{\lambda^{2}-b}\right) & (\lambda>\mu), \\ \left(n+\frac{1}{2}\right) \pi-\tan ^{-1}\left(\frac{\lambda^{2}-b}{a \lambda}\right) & (\lambda<\mu),\end{cases}
$$

where

$$
\mu=\frac{1}{2}\left[a+\sqrt{ }\left(a^{2}+4 b\right)\right] .
$$

Thus, when $n$ is large, $\lambda \approx n \pi$. This is the first approximation.

Note that if $a=0$, then $b=\mu=0$. In this case $\lambda \geq \mu$ and the eigenvalues are $\lambda=n \pi$, from (5.5) below. If $a \neq 0$ but $b=0$, then $\mu=a$, and $\lambda \tan \lambda=a$. The eigenvalues for this case are determined by (5.3), (5.4) for $\lambda<\mu$ and by (5.5) for $\lambda \geq \mu$.

(a) $\lambda<\mu$.

In this case $\left|a \lambda /\left(\lambda^{2}-b\right)\right|>1$. Some further consideration shows there are two intermediate regions of expansion depending upon the parameter $a \lambda / b$.

$$
\lambda<\mu, \frac{a \lambda}{b} \leq 1, a \neq 0, b \neq 0 .
$$

Since $a \lambda / b \leq 1$ and $4 b \leq a^{2}$, we have the inequality $\lambda^{2} \leq \frac{1}{4} b$,

$$
\begin{aligned}
\lambda_{n} & =q-(b-a)\left(\frac{1}{a^{2}}-\frac{1}{3 b}\right)\left(\frac{a q}{b}\right)^{3} \\
& +\left[(b-a)\left(\frac{1}{a^{2}}-\frac{1}{3 b}-\frac{b}{a^{4}}\right)+\frac{2}{15}\right]\left(\frac{a q}{b}\right)^{5}+O\left[\left(\frac{a q}{b}\right)^{7}\right],
\end{aligned}
$$




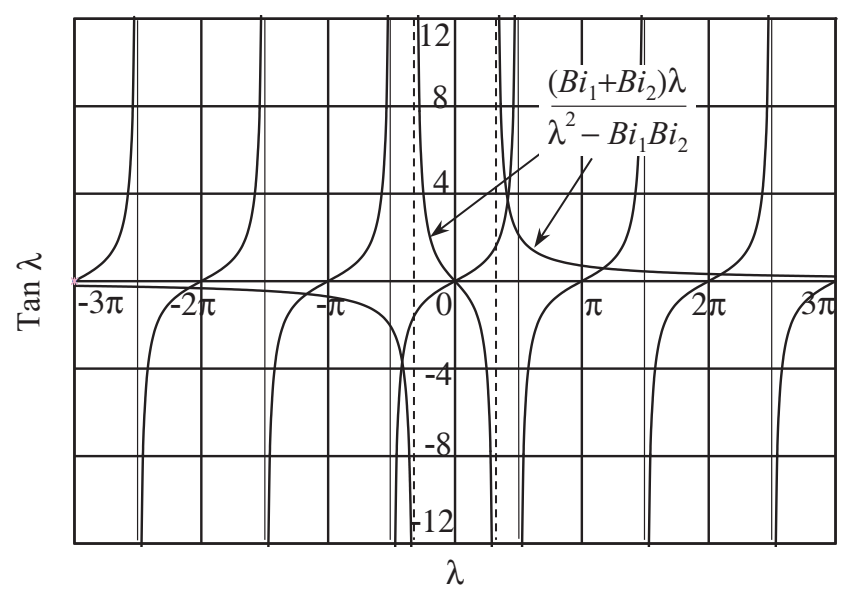

FIG. 2. Roots of $\tan \lambda=\left(B i_{1}+B i_{2}\right) \lambda /\left(\lambda^{2}-B i_{1} B i_{2}\right)$, for $B i_{1}=1$, $B i_{2}=1$. The dashed lines are $\lambda= \pm \sqrt{ }\left(B i_{1} B i_{2}\right)$.

where $q=(n+1) \pi b /(a+b), n=0,1,2, \ldots$

$$
\begin{gathered}
\lambda<\mu, \frac{a \lambda}{b}>1, a \neq 0 . \\
\lambda_{0}=\frac{\pi a}{4(a+1)}\left[1+\left\{\frac{16 b(a+1)}{\pi^{2} a^{2}}+1\right\}^{\frac{1}{2}}\right], \\
\lambda_{n}=r\left(1-\frac{1}{a}+\frac{1}{a^{2}}\right)+\frac{b}{a r}\left[1+\frac{1}{a^{2}}-\frac{b}{a r^{2}}\left(1+\frac{2}{a}\right)\right]+\frac{1}{3}\left[\frac{r}{a}\left(1-\frac{1}{a}\right)\right. \\
\left.-\frac{b}{a r}\left(1-\frac{b}{a r}\right)\right]^{3}-\frac{1}{5}\left[\frac{r}{a}\left(1-\frac{1}{a}\right)-\frac{b}{a r}\left(1-\frac{b}{a r}\right)\right]^{5} \\
+O\left[\left(\frac{r}{a}\right)^{7}\right]+O\left[\left(\frac{b}{a r}\right)^{7}\right], \quad(n>0),
\end{gathered}
$$

where $r=\left(n+\frac{1}{2}\right) \pi, n=1,2,3, \ldots$.

(b) $\lambda \geq \mu$.

In this case $\left|a \lambda /\left(\lambda^{2}-b\right)\right|<1$. Now $\lambda \geq \mu$ means $\lambda \geq \frac{1}{2} a\left[1+\sqrt{ }\left(1+4 b / a^{2}\right)\right]$, so that $a^{2} \leq \lambda^{2}$. Combining this with $4 b^{2} \leq a^{2}$, we get $4 b \leq \lambda^{2}$.

$$
\begin{aligned}
\lambda_{0}= & \tan ^{-1}\left[\frac{1}{3}(a+b)(b+3)(a+b+3)\right]^{\frac{1}{2}}, \\
\lambda_{n}= & p+\frac{a}{p}-a\left(a-b+\frac{1}{3} a^{2}\right) \frac{1}{p^{3}} \\
& +a\left(2 a^{2}+b^{2}-4 a b-a^{2} b+\frac{4}{3} a^{3}+\frac{1}{5} a^{4}\right) \frac{1}{p^{5}}+O\left(\frac{1}{p^{7}}\right) \quad(n>0),
\end{aligned}
$$

where $p=n \pi, n=1,2,3, \ldots$. Here principal values of inverse functions are to be taken. 
From (5.5) we have $\lambda_{n}-\lambda_{n-1}=\pi+O(1 / n)$ as $n \rightarrow \infty$. Thus, for large $n$, two consecutive roots are separated by $\pi$ nearly. Numerical results indicate that the difference $\lambda_{n}-\lambda_{n-1}$ approaches $\pi$ logarithmically slowly. In generating the eigenvalues the separation property may be used to verify that no eigenvalue was missed. From (3.4b) the Fourier coefficient $A_{n} \approx 1 /(n \pi)$ as $n \rightarrow \infty$. The order term suggests that series summation, in general, will converge slowly.

Indexing the eigenvalues. For $n=0$ let $\lambda_{0}$ be the lowest eigenvalue, for $n=1, \lambda_{1}$ the next higher eigenvalue, and so on. Then $n$ may be used as a counter to designate a sequence for the eigenvalues. Thus the eigenvalues are enumerated. For a given $n$ it is possible to determine a priori the regions of validity of formulas (5.3)-(5.5). If $a=0$, the eigenvalues are $\lambda=n \pi$. Otherwise, let $M=\operatorname{round}[(a+b) /(\pi a)-1], N=\operatorname{round}[\mu / \pi]$, where round $[x]$ is the integer nearest to $x$. Then use (5.3) for $n \leq M$, (5.4) for $M<n<$ $N$, and (5.5) for $n \geq N$. As an example, let $a=35, b=250$, so that $M=2, N=13$. Then starting with $n=0$, use (5.3) for $n \leq 2$, (5.4a-b) for $2<n<13$, and (5.5) for all $n \geq 13$. As another example, let $a=2, b=1$. Then $M=-1, N=1$ and we use (5.4) for $n=0$ and (5.5) for $n \geq 1$.

6. Eigenvalues: the trigonometric solution. Yet another solution may be given for the eigenvalues. Let $\tan \alpha=B i_{1} / \lambda, \tan \beta=B i_{2} / \lambda$. Then

$$
\tan (\alpha+\beta)=\frac{\left(B i_{1}+B i_{2}\right) \lambda}{\lambda^{2}-B i_{1} B i_{2}}=\tan \lambda, \quad \text { from }(5.1)
$$

Therefore,

$$
\begin{aligned}
\lambda & = \pm n \pi+\alpha+\beta \\
& = \pm n \pi+\tan ^{-1} \frac{B i_{1}}{\lambda}+\tan ^{-1} \frac{B i_{2}}{\lambda}, \quad n=0,1,2, \ldots .
\end{aligned}
$$

Linearizing the right-hand side with $\lambda_{n} \approx \bar{\lambda}_{n}= \pm\left(n+\frac{1}{2}\right) \pi$, the roots of (5.1) are given approximately as

$$
\lambda_{n} \approx \pm n \pi+\tan ^{-1} \frac{B i_{1}}{\bar{\lambda}_{n}}+\tan ^{-1} \frac{B i_{2}}{\bar{\lambda}_{n}}, \quad n=0,1,2, \ldots
$$

Here principal values of inverse functions will be taken. By selecting only the positive signs, eigenvalues of (5.1) are obtained. This is the simplest solution to the general eigenvalue problem. Equation (6.2) was tested over a wide range of Biot numbers. Although the accuracy is not as sharp as with the asymptotic solutions, it has the advantage that with one formula one generates a sequence of $\lambda_{n}$ in the neighborhood of the correct roots. When used as initial approximations in Newton's (or Householder's) formula the iterative process converges rapidly and is numerically stable. Although it is possible to improve the trigonometric solution further we shall not pursue this here. 
Special cases. When one of the Biot numbers, say $B i_{2}$, is zero, the eigenvalues satisfy $\lambda_{n} \tan \lambda_{n}=B i_{1}$. Another simplification results when the Biot numbers are equal. Let $B i_{1}=B i_{2}=B i$. Then from (6.1) eigenvalues are the roots of

$$
\tan \left(\frac{1}{2} \lambda_{n}-\frac{1}{2} n \pi\right)=\frac{B i}{\lambda_{n}}
$$

When $n$ is even, say $n=2 m, m=0,1,2, \ldots$, we get $\frac{1}{2} \lambda_{n} \tan \frac{1}{2} \lambda_{n}=\frac{1}{2} B i$. When $n$ is odd, say $n=2 m+1, m=0,1,2, \ldots$, we get $\frac{1}{2} \lambda_{n} \cot \frac{1}{2} \lambda_{n}=-\frac{1}{2} B i$. Special solutions of the two transcendental equations are given in Appendix A although (6.2) will also give the full solution for any combination of Biot numbers.

Higher accuracy. The asymptotic or the trigonometric solution gives the initial approximation. Increased accuracy is achieved by using Newton-Raphson, Householder, or other iterative methods. In its simplest form the Householder method for $p=1$ (cubic convergence) is,

$$
x_{i+1}=x_{i}-\frac{f\left(x_{i}\right)}{f^{\prime}\left(x_{i}\right)}\left\{1+\frac{f\left(x_{i}\right) f^{\prime \prime}\left(x_{i}\right)}{2\left[f^{\prime}\left(x_{i}\right)\right]^{2}}\right\} .
$$

Here

$$
f\left(x_{i}\right)=\tan x_{i}-\left(B i_{1}+B i_{2}\right) x_{i} /\left(x_{i}^{2}-B i_{1} B i_{2}\right)
$$

is the residual error. If the bracketed term \{\} in (6.3) is set to unity, it becomes Newton's method (quadratic convergence). We found Householder's algorithm only marginally superior to Newton's method. We calculated the first 1000 eigenvalues for a wide range of Biot numbers and found the method rapidly convergent. As an illustration the first ten eigenvalues for $B i_{1}=1, B i_{2}=1(a=2, b=1)$ are listed in Table 1. The accuracy shown is typical, especially when $n$ is large.

7. Steady state temperatures. From $(3.4 \mathrm{a}-\mathrm{c})$ the steady state temperature is

$$
T\left(z^{\prime}\right)=T_{1}+c\left(1+B i_{1} z^{\prime}\right)\left(T_{2}-T_{1}\right)
$$

where $c$ is a constant defined in (3.4c) and $z^{\prime}=z / L$. The temperature is linear in $z^{\prime}$. The average temperature $T_{a v}$ is at $z=\frac{1}{2} L$ and temperature difference between the inner and outer faces is $\Delta T=c B i_{1}\left(T_{1}-T_{2}\right)$. The heat flow per unit area through the plate is

$$
q_{1-2}=\kappa\left(\frac{1}{B i_{1}}+\frac{1}{B i_{2}}+1\right)^{-1} \frac{\left(T_{1}-T_{2}\right)}{L} .
$$

Steady state temperature with constant heat flux at the boundary. An important case arises when the ends at $z=0$ and $z=L$ receive constant heat fluxes $q_{1}$ and $q_{2}$, respectively. From an inspection of the boundary conditions (cf. Eq. (8.1) below) it is clear that the steady state solution may be obtained by substituting $T_{i}+q_{i} / h_{i}$ for $T_{i}$ in (7.1). Thus

$$
T\left(z^{\prime}\right)=T_{1}+\frac{q_{1}}{h_{1}}+c\left(T_{2}-T_{1}+\frac{q_{2}}{h_{2}}-\frac{q_{1}}{h_{1}}\right)\left(1+B i_{1} z^{\prime}\right) \quad\left(h_{1}, h_{2} \neq 0\right),
$$


TABLE 1. First 10 roots of $\tan \lambda=\left(B i_{1}+B i_{2}\right) \lambda /\left(\lambda^{2}-B i_{1} B i_{2}\right)$,
$B i_{1}=1, B i_{2}=1 . \quad \lambda_{n}=n \pi+O(1 / n), n \rightarrow \infty$.
Residual error $R_{n}=\tan \lambda_{n}-\left(B i_{1}+B i_{2}\right) \lambda_{n} /\left(\lambda_{n}^{2}-B i_{1} B i_{2}\right)$.

\begin{tabular}{c|c|c|c|c||c|c}
\hline & \multicolumn{2}{|c||}{$\begin{array}{c}\text { Asymptotic solution } \\
\text { Eqs. }(5.3)-(5.5)\end{array}$} & \multicolumn{2}{c||}{$\begin{array}{c}\text { Trigonometric solution } \\
\text { Eq. }(6.2)\end{array}$} & \multicolumn{2}{c}{$\begin{array}{c}\text { With iterative improvement } \\
\left|R_{n}\right|<10^{-14}\end{array}$} \\
\hline$n$ & $\lambda_{n}$ & $R_{n}$ & $\lambda_{n}$ & $R_{n}$ & $\lambda_{n}$ & $\lambda_{n}-\lambda_{n-1}$ \\
\hline 0 & 1.3030 & $-9.0 \times 10^{-2}$ & 1.1338 & $-5.8^{a}$ & 1.306542374189 & - \\
1 & 3.6987 & $4.0 \times 10^{-2}$ & 3.5598 & $-1.7 \times 10^{-1}$ & 3.673194406304 & 2.3667 \\
2 & 6.5849 & $3.2 \times 10^{-4}$ & 6.5365 & $-5.4 \times 10^{-2}$ & 6.584620042564 & 2.9114 \\
3 & 9.6317 & $1.9 \times 10^{-5}$ & 9.6062 & $-2.7 \times 10^{-2}$ & 9.631684635692 & 3.0471 \\
4 & 12.7232 & $2.5 \times 10^{-6}$ & 12.7076 & $-1.6 \times 10^{-2}$ & 12.723240784131 & 3.0916 \\
5 & 15.8341 & $5.3 \times 10^{-7}$ & 15.8236 & $-1.1 \times 10^{-2}$ & 15.834105369332 & 3.1109 \\
6 & 18.9550 & $1.5 \times 10^{-7}$ & 18.9474 & $-7.7 \times 10^{-3}$ & 18.954971410842 & 3.1209 \\
7 & 22.0817 & $5.1 \times 10^{-8}$ & 22.0760 & $-5.7 \times 10^{-3}$ & 22.081659635943 & 3.1267 \\
8 & 25.2120 & $2.0 \times 10^{-8}$ & 25.2076 & $-4.5 \times 10^{-3}$ & 25.212026888551 & 3.1304 \\
9 & 28.3449 & $8.7 \times 10^{-9}$ & 28.3413 & $-3.6 \times 10^{-3}$ & 28.344864149600 & 3.1328 \\
\hline
\end{tabular}

a Newton's method converged even though the residual is large. The residual error was $1.4 \times 10^{-8}$ after four iterations, and $<10^{-16}$ after one extra iteration.

or,

$$
\theta=\frac{T-T_{1}}{T_{2}-T_{1}}=\frac{q_{1}^{\prime}}{B i_{1}}+c\left(1+\frac{q_{2}^{\prime}}{B i_{2}}-\frac{q_{1}^{\prime}}{B i_{1}}\right)\left(1+B i_{1} z^{\prime}\right) \quad\left(B i_{1}, B i_{2} \neq 0\right)
$$

where $q_{i}^{\prime}=q_{i} L /\left[\kappa\left(T_{2}-T_{1}\right)\right], i=1$ or 2 , is a nondimensional heat flux parameter. The artifice fails when one or both Biot numbers vanish. This case is discussed in the next section. Parametric variation of $\theta$ with $B i_{1}, B i_{2}$ for any given $q_{1}^{\prime}, q_{2}^{\prime}$ is obtained from (7.2). For the space-based mirror in our example, if $T_{1}=293^{\circ} \mathrm{K}, T_{2}=5^{\circ} \mathrm{K}, \epsilon_{2} \mathcal{F}_{2}=0.015$, $L=10 \mathrm{~cm}$, then from (2.3) and Table $2, h_{2}=0.022 \mathrm{~W} /\left(\mathrm{m}^{2} \mathrm{~K}\right)$ and $B i_{2}=0.0014$. By comparison, $h_{1}=4.85 \mathrm{~W} /\left(\mathrm{m}^{2} \mathrm{~K}\right), B i_{1}=0.3$, for $\epsilon_{1} \mathcal{F}_{1}=0.85$.

8. Combined radiation and heat flux at the boundary. The modified end conditions are (cf. $(2.1 \mathrm{c}-\mathrm{d}))$

$$
\left.\begin{array}{rl}
\kappa \frac{\partial T}{\partial z} & =h_{1}\left(T-T_{1}\right)-q_{1}, \quad z=0, t>0, \\
-\kappa \frac{\partial T}{\partial z} & =h_{2}\left(T-T_{2}\right)-q_{2}, \quad z=L, t>0 .
\end{array}\right\}
$$


For now we assume the fluxes to be constant. To reduce the end conditions homogeneous we use the linear transformation $T=a_{0}^{\prime}+b_{0}^{\prime} z+\varphi$, and choose the coefficients as

$$
\begin{array}{r}
a_{0}^{\prime}=T_{1}+\frac{q_{1}}{h_{1}}+c\left(T_{2}-T_{1}+\frac{q_{2}}{h_{2}}-\frac{q_{1}}{h_{1}}\right), \quad b_{0}^{\prime} L=c B i_{1}\left(T_{2}-T_{1}+\frac{q_{2}}{h_{2}}-\frac{q_{1}}{h_{1}}\right), \\
\left(B i_{1} . B i_{2} \neq 0\right) .
\end{array}
$$

If $B i_{1}=0$, then $a_{0}^{\prime}=T_{2}+\frac{q_{2}}{h_{2}}+\frac{q_{1}}{h_{2}}\left(1+B i_{2}\right), \quad b_{0}^{\prime} L=-\frac{q_{1} L}{\kappa}$.

If $B i_{2}=0$, then $a_{0}^{\prime}=T_{1}+\frac{q_{1}+q_{2}}{h_{1}}, \quad b_{0}^{\prime} L=\frac{q_{2} L}{\kappa}$.

The temperature solution is

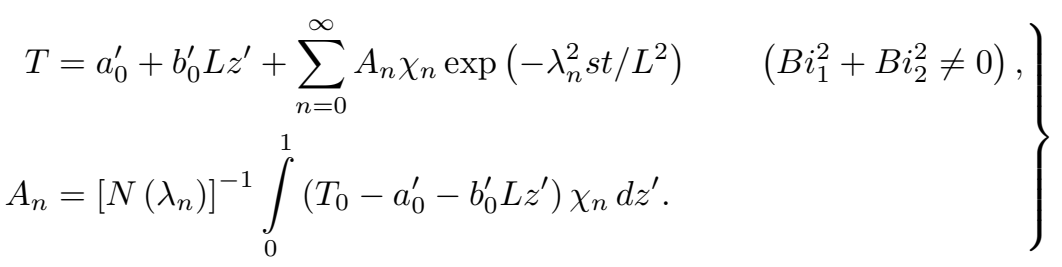

The eigenfunction $\chi_{n}$ and the square of the norm $N\left(\lambda_{n}\right)$ are given in (3.1), (3.3), and the eigenvalues $\lambda_{n}$ satisfy (3.5). If $q_{1}=q_{2}=0$, we recover (3.4a-c).

One must be careful in using the formal solutions if heat flux is specified at every point of the boundary. The problem, in general, may not have a steady state solution. Consider, for example, the case of $B i_{1}=B i_{2}=0$. In this case the linear transformation fails. This is because when the plate is insulated at both ends, no steady state is possible unless the incoming heat is exactly balanced by the heat absorbed by its mass. This is analogous to water being boiled in a beaker. After the water has fully evaporated the temperature of the container will keep on rising. By applying Gauss' divergence theorem to (2.1a) it is seen that in the steady state, and in the absence of heat generation, that

$$
\sum_{S=\partial V}\left(\kappa \frac{\partial T}{\partial n}\right)_{i}=0
$$

The end conditions may not satisfy this equation and therefore no steady state solution is possible. In such case we use the transformation $T=\beta t+b_{0}^{\prime \prime} z+c_{0}^{\prime \prime} z^{2}+\varphi$ [6], p. 201, where

$$
\beta=\frac{s}{\kappa V} \iint_{S=\partial V} q_{i} d S
$$

and

$$
b_{0}^{\prime \prime} L=-\frac{q_{1} L}{\kappa}, \quad c_{0}^{\prime \prime} L^{2}=\frac{1}{2}\left(\frac{q_{1} L}{\kappa}+\frac{q_{2} L}{\kappa}\right),
$$


which makes the end conditions homogeneous. Eigenfunctions of the homogeneous system are $\cos \left(n \pi z^{\prime}\right), n=0,1,2, \ldots$. The temperature solution is

$$
\left.\begin{array}{rl}
T= & 2\left(c_{0}^{\prime \prime} L^{2}\right) \frac{s t}{L^{2}}+b_{0}^{\prime \prime} L z^{\prime}+c_{0}^{\prime \prime} L^{2} z^{2} \\
& +A_{0}+\sum_{n=1}^{\infty} A_{n} \cos \left(n \pi z^{\prime}\right) \exp \left(-n^{2} \pi^{2} s t / L^{2}\right), \\
A_{0}= & -\frac{1}{2} b_{0}^{\prime \prime} L-\frac{1}{3} c_{0}^{\prime \prime} L^{2}+\int_{0}^{1} T_{0} d z^{\prime}, \\
A_{n}= & 2 \int_{0}^{1}\left[T_{0}-b_{0}^{\prime \prime} L z^{\prime}-c_{0}^{\prime \prime} L^{2} z^{\prime 2}\right] \cos \left(n \pi z^{\prime}\right) d z^{\prime} \quad(n>1) .
\end{array}\right\}
$$

\section{Transient temperature response.}

Radiation boundary condition. We show in Fig. 3(a) transient temperature response for $B i_{1}=1$ and $B i_{2}=0.1,0.5,1,5,10,20, \infty$. The initial temperature is taken as $T_{0}=T_{1}$. In each case the maximum temperature is reached asymptotically at large Fourier numbers. Temperature distribution through the thickness is shown in Fig. 3(b) for $B i_{1}=1, B i_{2}=10$. The trends are similar at other Biot numbers.

\section{Recovery of the constant boundary condition and other solutions.}

Mixed boundary conditions. By allowing the Biot numbers to go to infinity a constant boundary temperature is specified. Thus, the formulation and the results given above apply to a wide class of boundary conditions. We elucidate this with an example of mixed boundary conditions when the surface at $z=0$ is held at constant temperature $T_{1}$, but the surface at $z=L$ radiates to space at $T_{2}$. We set $B i_{1}=\infty$ in $(3.4 \mathrm{a}-\mathrm{c})$ and obtain

$$
\left.\begin{array}{c}
\theta=\frac{T-T_{1}}{T_{2}-T_{1}}=\frac{B i_{2}}{1+B i_{2}} z^{\prime}+\sum_{n=0}^{\infty} A_{n} \sin \left(\lambda_{n} z^{\prime}\right) \exp \left(-F o \lambda_{n}^{2}\right) \quad\left(T_{1} \neq T_{2}\right), \\
A_{n}=2 \frac{\lambda_{n}^{2}+B i_{2}^{2}}{\lambda_{n}^{2}+B i_{2}^{2}+B i_{2}} \int_{0}^{1}\left\{\frac{T_{0}-T_{1}}{T_{2}-T_{1}}-\frac{B i_{2}}{1+B i_{2}} z^{\prime}\right\} \sin \left(\lambda_{n} z^{\prime}\right) d z^{\prime} .
\end{array}\right\}
$$

The eigenvalues are nonnegative roots of $\lambda_{n} \cot \lambda_{n}=-B i_{2}, B i_{2} \geq 0$ (see Appendix A for the solution). Further simplification occurs when $T_{1}=T_{2}$. Other mixed boundary value problems are solved in a similar manner. 


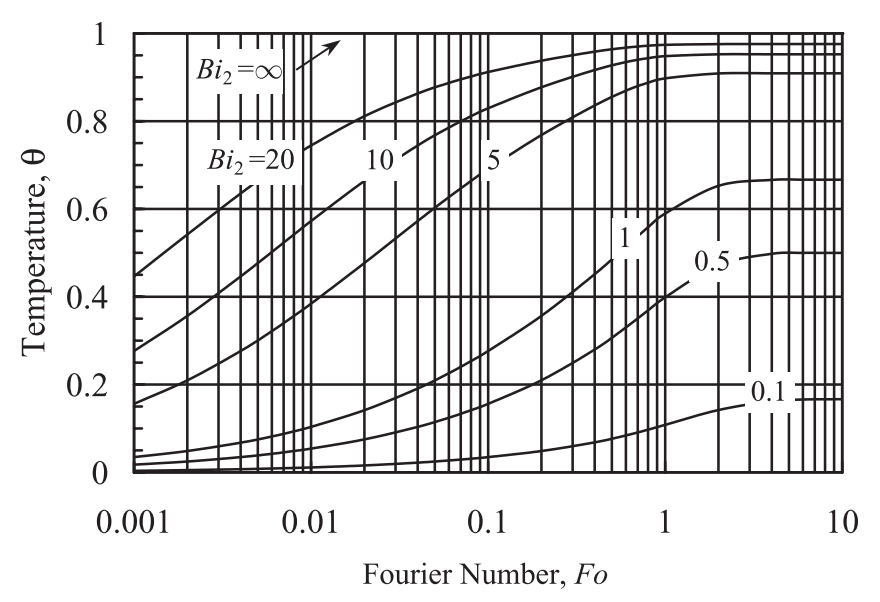

FIG. 3(a). Transient temperature response of a free plate with unequal boundary temperatures and unequal heat transfer rates at endpoints. $\theta=\left(T-T_{1}\right) /\left(T_{2}-T_{1}\right), T_{0}=T_{1}, B i_{1}=1$. Surface temperature at $z=L . B i_{2}=0.1,0.5,1,5,10,20, \infty$. As $F_{o} \rightarrow \infty$, $\theta \rightarrow 2 B i_{2} /\left(1+2 B i_{2}\right)$.

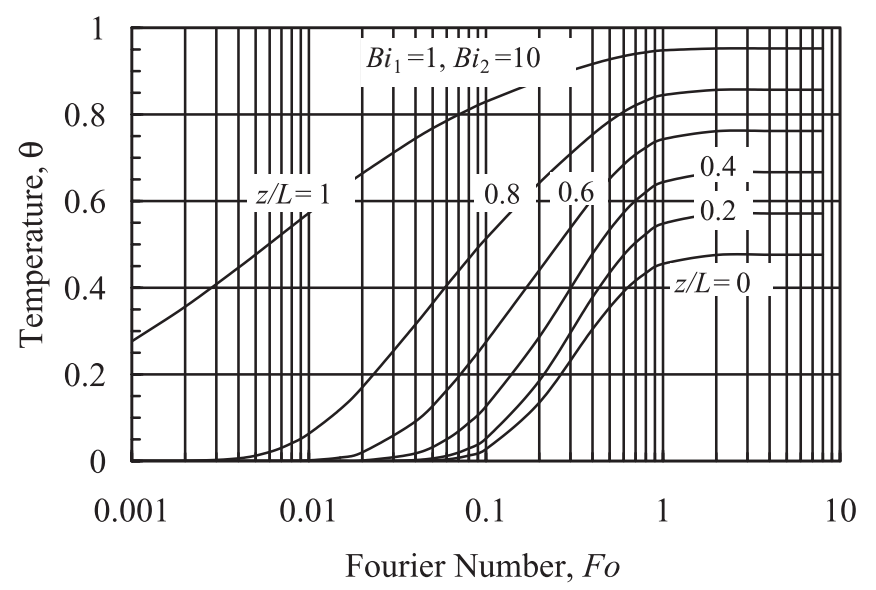

FIG. 3(b). Transient temperature response of a free plate with unequal boundary temperatures and unequal heat transfer rates at endpoints. $\theta=\left(T-T_{1}\right) /\left(T_{2}-T_{1}\right), T_{0}=T_{1}, B i_{1}=1$. Temperature through the thickness: $B i_{2}=10, z / L=0,0.2,0.4,0.6,0.8,1$. As $F o \rightarrow \infty, \theta \rightarrow c(1+z / L), c=10 / 21$.

Constant temperature conditions. $B i_{1}=\infty, B i_{2}=\infty$, and end temperatures are specified:

$$
\left.\begin{array}{rl}
\theta & =\frac{T-T_{1}}{T_{2}-T_{1}}=z^{\prime}+\sum_{n=1}^{\infty} A_{n} \sin \left(n \pi z^{\prime}\right) \exp \left(-F o n^{2} \pi^{2}\right) \quad\left(T_{1} \neq T_{2}\right), \\
A_{n} & =2 \int_{0}^{1}\left[\frac{T_{0}-T_{1}}{T_{2}-T_{1}}-z^{\prime}\right] \sin \left(n \pi z^{\prime}\right) d z^{\prime} .
\end{array}\right\}
$$

If $T_{1}=T_{2}$, then the solution is simplified further. 


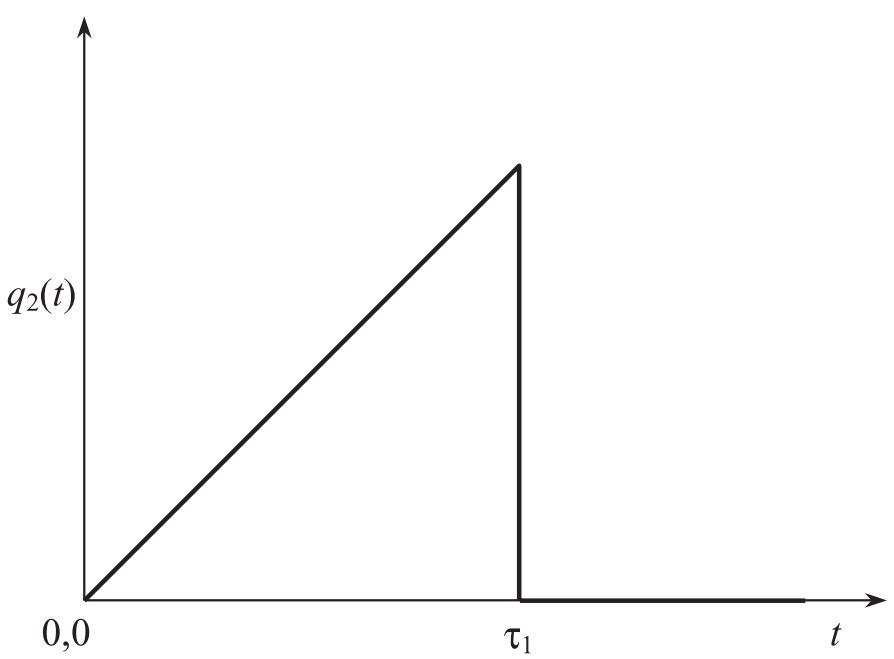

Fig. 4. Profile of a time-varying heat flux, $q_{2}(t)=\gamma t$.

Radiation condition with time-varying heat flux at the boundary. During science observation, a spacecraft's mirror will be exposed to a time-varying heat flux from space. This case is of considerable interest because as the mirror is being tilted through small angles to focus from one star to another, the incoming heat flux on the exposed face of the mirror $(z=L)$ will vary with time. Because of this an optical image distortion will occur. For precision control it is necessary to calculate the mirror's thermal deformation and to establish that the optical distortion error is within the allowable budget. The solution obtained in the previous sections is easily extended to include a time-varying heat flux by the use of Duhamel's principle [2], 8, [21. This may be done in two ways. In one approach an equivalent boundary temperature $T_{2}(t)$ at $z=L$ will be specified; no additional heat flux needs to be specified as its effect is already included in $T_{2}(t)$. Or equivalently, a boundary temperature and a heat flux $q_{2}(t)$ at $z=L$ will be specified. Here we follow the second approach. We start with the differential equation system $(2.1 \mathrm{a}-\mathrm{d})$ transformed from $T$ to $\varphi$. The modified end condition is

$$
-\kappa \frac{\partial \varphi}{\partial z}-h_{2} \varphi=-q_{2}(t), \quad z=L, \quad t>0 .
$$

Let us assume the mirror is rotated for the time $\tau_{1}$ before coming to a stop. It is reasonable to assume the heat flux to be represented by

$$
q_{2}(t)= \begin{cases}\gamma t & \left(t<\tau_{1}\right), \\ 0 & \left(t>\tau_{1}\right),\end{cases}
$$

where $\gamma$ is some constant; see Fig. 4. To give some perspective, if $\gamma=1 \mathrm{~W} /\left(\mathrm{m}^{2} \mathrm{~s}\right)$ and $\tau_{1}=100 \mathrm{~s}$, then on average the incoming heat flux is $50 \mathrm{~W} / \mathrm{m}^{2}$. For a mirror pointed to deep space (anti-sun side) chief sources of the incoming heat flux are the starlights and background infrared radiation from the spacecraft itself. Total external heat flux, in all practicality, will be a fraction of this value. 
The solution is obtained using superposition. For homogeneous end conditions and a nonhomogeneous initial condition, an auxiliary solution $\Phi_{1}\left(z^{\prime}, t\right)$ is

$$
\left.\begin{array}{l}
\Phi_{1}\left(z^{\prime}, t\right)=\left(T_{2}-T_{1}\right) \sum_{n=0}^{\infty} A_{n} \chi_{n} \exp \left(-\lambda_{n}^{2} s t / L^{2}\right), \\
A_{n}=\left[N\left(\lambda_{n}\right)\right]^{-1} \int_{0}^{1}\left[\frac{T_{0}-T_{1}}{T_{2}-T_{1}}-c\left(1+B i_{1} z^{\prime}\right)\right] \chi_{n} d z^{\prime} .
\end{array}\right\}
$$

The eigenfunction $\chi_{n}$, and the square of the norm $N\left(\lambda_{n}\right)$, are given in Eqs. (3.1), (3.3), and the eigenvalues $\lambda_{n}$ satisfy (3.5). A second auxiliary solution $\Phi\left(z^{\prime}, t\right)$ is obtained by setting $q_{2}(t)=1$ in Eq. (9.3) and keeping other boundary conditions homogeneous,

$$
\left.\begin{array}{l}
\Phi\left(z^{\prime}, t\right)=\frac{c}{h_{2}}\left(1+B i_{1} z^{\prime}\right)+\frac{c}{h_{2}} \sum_{n=0}^{\infty} A_{n}^{\prime} \chi_{n} \exp \left(-\lambda_{n}^{2} s t / L^{2}\right), \\
A_{n}^{\prime}=-\left[N\left(\lambda_{n}\right)\right]^{-1} \int_{0}^{1}\left(1+B i_{1} z^{\prime}\right) \chi_{n} d z^{\prime} .
\end{array}\right\}
$$

Duhamel's principle gives the solution for the time-varying boundary condition (9.3) as

$$
\Phi_{2}\left(z^{\prime}, t\right)=\frac{\partial}{\partial t} \int_{0}^{t} q_{2}(\tau) \Phi\left(z^{\prime}, t-\tau\right) d \tau=-\int_{0}^{t} q_{2}(\tau) \frac{\partial \Phi\left(z^{\prime}, t-\tau\right)}{\partial \tau} d \tau
$$

since $\partial \Phi\left(z^{\prime}, t-\tau\right) / \partial t=-\partial \Phi\left(z^{\prime}, t-\tau\right) / \partial \tau$. The solution is

$$
\begin{aligned}
\Phi_{2}\left(z^{\prime}, t\right)= & \int_{0}^{t} \frac{d q_{2}(\tau)}{d \tau}\left\{\frac{c}{h_{2}}\left(1+B i_{1} z^{\prime}\right)+\frac{c}{h_{2}} \sum_{n=0}^{\infty} A_{n}^{\prime} \chi_{n} \exp \left[-\lambda_{n}^{2} s(t-\tau) / L^{2}\right]\right\} d \tau \\
= & \frac{c \gamma t}{h_{2}}\left(1+B i_{1} z^{\prime}\right)+\frac{c \gamma L^{2}}{s h_{2}} \sum_{n=0}^{\infty} A_{n}^{\prime} \frac{\chi_{n}}{\lambda_{n}^{2}}\left[1-\exp \left(-F o \lambda_{n}^{2}\right)\right], \quad t<\tau_{1}, \\
\Phi_{2}\left(z^{\prime}, t\right)= & \frac{c \gamma L^{2}}{s h_{2}} \sum_{n=0}^{\infty} A_{n}^{\prime} \frac{\chi_{n}}{\lambda_{n}^{2}}\left\{\exp \left[\left(-F o+F o^{\prime}\right) \lambda_{n}^{2}\right]-\exp \left(-F o \lambda_{n}^{2}\right)\right\} \\
& -\frac{c \gamma \tau_{1}}{h_{2}} \sum_{n=0}^{\infty} A_{n}^{\prime} \chi_{n} \exp \left[\left(-F o+F o^{\prime}\right) \lambda_{n}^{2}\right], \quad t>\tau_{1}
\end{aligned}
$$

Here $F o=s t / L^{2}, F o^{\prime}=s \tau_{1} / L^{2}$. The continuity at $t=\tau_{1}$ is assured by noting that

$$
1+B i_{1} z^{\prime}=-\sum_{n=0}^{\infty} A_{n}^{\prime} \chi_{n}
$$

In practice, the second series in (9.7b) is found to converge slowly at small Fourier numbers. There are methods to improve the convergence rate for "small times" which will not be discussed here. Finally, the desired solution is obtained by adding the auxiliary 
solutions $\Phi_{1}$ and $\Phi_{2}$ and then transforming back to $T\left(z^{\prime}, t\right)$,

$$
\theta=\frac{T-T_{1}}{T_{2}-T_{1}}=c\left(1+B i_{1} z^{\prime}\right)+\left(T_{2}-T_{1}\right)^{-1} \Phi_{1}\left(z^{\prime}, t\right)+\left(T_{2}-T_{1}\right)^{-1} \Phi_{2}\left(z^{\prime}, t\right) .
$$

As a check, if $\gamma=0, \Phi_{2}=0$ and solution $(3.4 \mathrm{a}-\mathrm{c})$ is recovered.

10. Thermoelastic equations. A common engineering practice is to compute thermal stresses in an elastic body by solving first the temperature from Fourier's heat conduction equation, and then the stresses from Navier's equations. Considerable investigation has been done to determine the conditions under which such uncoupling of the governing equations is valid. When the nonuniform temperature distribution is primarily due to the heat supplied to the body from external sources, as it is in this paper, the mechanical coupling terms in the energy balance equation may be neglected in comparison to the thermal terms if the heating rate is slow. If, on the other hand, temperature differences are due solely to the deformation of the body, like the continuous bending of a wire, then the coupled problem must be considered [6]. For constant properties and in the absence of body force and heat generation, the coupled linear thermoelastic equations are

$$
\left.\begin{array}{c}
\kappa \nabla^{2} \vartheta=\rho c \frac{\partial \vartheta}{\partial t}+T_{0} \alpha(3 \lambda+2 \mu) \nabla \cdot \dot{\boldsymbol{u}}, \\
\boldsymbol{u}+(\lambda+\mu) \nabla(\nabla \cdot \boldsymbol{u})=\alpha(3 \lambda+2 \mu) \nabla \vartheta+\rho \ddot{\boldsymbol{u}} .
\end{array}\right\}
$$

Equation (10.1a) is the generalized Fourier heat conduction equation and Eq. (10.1b) is the generalized Navier displacement-temperature equation. Here $\boldsymbol{u}$ is the displacement vector, $\vartheta=T-T_{0}$ is the temperature difference, and $T_{0}$ the temperature of a reference state of the body. All other notations have their usual meanings.

The Duhamel-Neumann form of Hooke's law for isotropic materials is

$$
\sigma_{i j}=\lambda \epsilon_{k k} \delta_{i j}+2 \mu \epsilon_{i j}-\alpha(3 \lambda+2 \mu) \vartheta \delta_{i j},
$$

where $\lambda$ and $\mu$ are the Lamé constants, $\alpha$ is the linear coefficient of thermal expansion, and $\delta_{i j}$ is the Kronecker delta. In addition, six Beltrami-Mitchell compatibility equations will have to be satisfied,

$$
(1+\nu) \nabla^{2} \sigma_{i j}+\sigma_{k k, i j}+\alpha E\left[\delta_{i j} \frac{1+\nu}{1-\nu} \nabla^{2} \vartheta+\vartheta_{, i j}\right]=0 .
$$

Carlson 7] develops thermoelastic equations from a rigorous treatment of the basic laws of thermomechanics. Biot [3] treats the problem from a consideration of the laws of irreversible thermodynamics. The uniqueness of the coupled thermoelastic problem is proved by Weiner [26. See also [19. Day [9, 10] has investigated uniqueness and consistency of the coupled one-dimensional thermoelastic problem. A more general thermoelastic formulation may be given, but a closed-form solution will not be simple (see, for example, Carlson [7, §20). 
TABLE 2. Thermal and elastic properties of glass

\begin{tabular}{ll}
\hline Mirror material & Glass $\left(\right.$ Zerodur $\left.^{\mathrm{TM}}\right)$ \\
Emissivity of the coating, $\epsilon$ & $\epsilon_{1}=0.85, \epsilon_{2}=0.015$ \\
Thermal conductivity, $\kappa$ & $1.6 \mathrm{~W} /(\mathrm{mK})$ \\
Density, $\rho$ & $2545 \mathrm{~kg} / \mathrm{m}^{3}$ \\
Young's modulus, $E$ & $90.3 \mathrm{GPa}$ \\
Poisson's ratio, $\nu$ & 0.24 \\
Thermal diffusivity, $s=\kappa /(\rho c)$ & $7.76 \times 10^{-7} \mathrm{~m}^{2} / \mathrm{s}$ \\
Coefficient of thermal expansion, $\alpha$ & $5.0 \times 10^{-8} /{ }^{\circ} \mathrm{K}$ \\
\hline
\end{tabular}

11. The uncoupled thermoelastic solution. We study the mirror as a free plate [6], 23] with radiation boundary conditions. With temperature varying only with $z$, and in the absence of body force and surface traction, it is reasonable to assume stress components of the form

$$
\sigma_{x x}=\sigma_{y y}=f(z) .
$$

From Saint-Venant's principle the solution is an accurate approximation for traction free edges at distances from edges larger than the plate thickness. The stress field satisfies the equilibrium equation (10.1b) identically, and the compatibility equations (10.3) reduce to

$$
\frac{\partial^{2} f}{\partial z^{2}}+\frac{\alpha E}{1-\nu}\left(\frac{\partial^{2} \vartheta}{\partial z^{2}}\right)=0
$$

Integrating this we obtain

$$
\sigma_{x x}=\sigma_{y y}=-\frac{\alpha E}{1-\nu} \vartheta+d_{1} z+d_{2} .
$$

Next, $d_{1}$ and $d_{2}$ are so chosen that the resultant force and momentum produced by the stresses vanish. This yields

$$
d_{1}=\frac{6}{(1-\nu) L}\left(-N_{T}+2 M_{T}\right), \quad d_{2}=\frac{2}{(1-\nu)}\left(2 N_{T}-3 M_{T}\right),
$$

where

$$
N_{T}=\alpha E \int_{0}^{1} \vartheta d z^{\prime}, \quad M_{T}=\alpha E \int_{0}^{1} \vartheta z^{\prime} d z^{\prime} .
$$

The strain components are obtained similarly and the shearing strains are zero. The displacement components are not fully determined by stresses and strains alone. For example, when the displacement-strain relation $\partial w / \partial z=\epsilon_{z z}$ is integrated we obtain

$$
w=-\frac{2 \nu}{E}\left(\frac{1}{2} d_{1} z^{2}+d_{2} z\right)+\frac{1+\nu}{1-\nu} \alpha \int_{0}^{z} \vartheta d z+w_{0}(x, y),
$$


and similarly for $u$ and $v$. By making full use of the constitutive relations (10.2), functional forms for $w_{0}(x, y)$ and others are obtained. The remaining constants of integration are then determined by applying zero displacement and zero rotation about the origin. The method is discussed fully in Timoshenko [23, $\S 100$.

The displacement components (exclusive of rigid body motion) are

$$
\left.\begin{array}{l}
\frac{u}{x}=\frac{v}{y}=\frac{1-\nu}{E}\left(2 d_{1} z+d_{2}\right), \\
w=-\frac{2 \nu}{E}\left(\frac{1}{2} d_{1} z^{2}+d_{2} z\right)+\frac{1+\nu}{1-\nu} \alpha \int_{0}^{z} \vartheta d z-\frac{1-\nu}{E}\left(x^{2}+y^{2}\right) d_{1} .
\end{array}\right\}
$$

The temperature difference $\vartheta$ is known from the previous sections. For simplicity we now assume the initial temperature $T_{0}$ to be constant and the reference state. From $(3.4 \mathrm{a}-\mathrm{c})$

$$
\vartheta=T_{1}-T_{0}+c\left(1+B i_{1} z^{\prime}\right)\left(T_{2}-T_{1}\right)+\left(T_{2}-T_{1}\right) \sum_{n=0}^{\infty} A_{n} \chi_{n} \exp \left(-\lambda_{n}^{2} s t / L^{2}\right) .
$$

Using this in (11.2) and (11.3), the terms $d_{1}, d_{2}$ reduce to

$$
\begin{aligned}
& d_{1}=\frac{\alpha E\left(T_{2}-T_{1}\right)}{(1-\nu) L}\left[c B i_{1}+6 \sum_{n=0}^{\infty} A_{n}\left(-J_{n}+2 K_{n}\right) \exp \left(-\lambda_{n}^{2} s t / L^{2}\right)\right], \\
& d_{2}=\frac{\alpha E\left(T_{2}-T_{1}\right)}{(1-\nu)}\left[\frac{T_{1}-T_{0}}{T_{2}-T_{1}}+c+2 \sum_{n=0}^{\infty} A_{n}\left(2 J_{n}-3 K_{n}\right) \exp \left(-\lambda_{n}^{2} s t / L^{2}\right)\right],
\end{aligned}
$$

with $A_{n}, \lambda_{n}$ from (3.4b), (3.5) and $J_{n}, K_{n}$ from (3.6). The results are simplified when the radiation sinks are equal $\left(T_{1}=T_{2}\right)$; cf. $(3.4 \mathrm{a}-\mathrm{c})$. With this, the stress and deformation of a radiatively cooled (or heated) free plate are fully determined. The curvature is

$$
\frac{1}{R_{x}} \approx \frac{\partial^{2} w}{\partial y^{2}}=-\frac{2(1-\nu)}{E} d_{1}, \quad \frac{1}{R_{y}} \approx \frac{\partial^{2} w}{\partial x^{2}}=-\frac{2(1-\nu)}{E} d_{1} .
$$

In the steady state,

$$
d_{1}=\frac{\alpha E}{(1-\nu) L} c B i_{1}\left(T_{2}-T_{1}\right), \quad d_{2}=\frac{\alpha E}{(1-\nu)}\left[T_{1}-T_{0}+c\left(T_{2}-T_{1}\right)\right],
$$

and

$$
\frac{1}{R_{x}}=\frac{1}{R_{y}} \approx-2 \alpha\left(\frac{1}{B i_{1}}+\frac{1}{B i_{2}}+1\right)^{-1}\left(\frac{T_{2}-T_{1}}{L}\right) .
$$

In the steady state the curvature is proportional to the heat flowing through the body. The use of the steady state values of $d_{1}, d_{2}$ in (11.1) results in zero stresses even though the curvature is nonzero, and concave up if $T_{2}<T_{1}$.

12. Discussion of the result. In the U.S., pioneering work carried out at Columbia, Brown, and Stanford from the early 1940s and through the 1960s has brought thermoelasticity to its present state [23, 6]. Because of the practical importance, transient temperature and heat flow rates in simple geometrical shapes have been calculated and results are presented in graphical charts for ready use; see [5], 16], [15], and especially 22. In this regard the unsteady conduction charts of Gröber or Heisler [14] or Boelter 
et al. [5] are well known. Due to the mathematical difficulties fewer solutions are available when thermal radiation is included, and compilation similar to 22 for thermal stresses is not available. Boley and Weiner [6] give a detailed treatment of the thermoelastic formulation, with examples of temperature and stresses in plates, cylinders, and spheres. Hlinka et al. [16] published charts on elastic thermal stresses for the heating and cooling of slabs and cylinders. The results are valid for $B i_{1}=B i_{2}$, and $q_{1}=q_{2}=0$. Heisler 14 presents the solution for the heating at constant temperature or constant heat flux. Zerkle and Sunderland 27] have published an extensive set of temperature response charts. The authors assume the plate insulated at one end $\left(B i_{1}=0\right)$, and the other end exchanging heat by radiation to medium at constant temperature. The ratio $T_{0} / T_{2}$ is used as a parameter indicative of the heating of the plate for $T_{0} / T_{2}<1$, and cooling for $T_{0} / T_{2}>1$. From (3.7) the solution to this problem for constant $T_{0}$ is

$$
\frac{T-T_{2}}{T_{0}-T_{2}}=2 \sum_{n=0}^{\infty} \frac{\sin \lambda_{n}}{\lambda_{n}+\sin \lambda_{n} \cos \lambda_{n}} \cos \left(\lambda_{n} z^{\prime}\right) \exp \left(-\lambda_{n}^{2} s t / L^{2}\right)
$$

so that the only truly independent parameter is the Biot number $B i_{2}$. In any case, the assumption of the initial temperature linearly proportional to the boundary temperature is restrictive and unnecessary. Here $\lambda_{n} \tan \lambda_{n}=B i_{2}$, and the series converges rapidly. The heat lost (or gained) per unit area by the plate from $t=0$ to $t=\tau$ is

$$
\frac{Q}{A}=\frac{2 \kappa L}{s}\left(T_{0}-T_{2}\right) \sum_{n=0}^{\infty} \frac{\sin ^{2} \lambda_{n}}{\lambda_{n}^{2}+B i_{2} \cos ^{2} \lambda_{n}}\left[1-\exp \left(-\lambda_{n}^{2} s \tau / L^{2}\right)\right] .
$$

For stress components substitute $\chi_{n}=\cos \left(\lambda_{n} z^{\prime}\right), A_{n}=2 \sin \lambda_{n} /\left(\lambda_{n}+\sin \lambda_{n} \cos \lambda_{n}\right)$ in (12.1) below. The strain-displacements are similarly obtained. These are special cases of the more general solution presented in this paper. Indeed, with the easy, accurate, and rapid determination of the eigenvalues, one-dimensional transient response of plates and spherical shells can be obtained with very little effort.

Radiation boundary conditions. We discuss here a select number of cases that are of general interest. The parameters in the examples are not necessarily representative of a space-based mirror. We consider a mirror exchanging heat by radiation to unequal boundary temperatures at unequal heat transfer rates. The temperature solution to this problem was given in $(3.4 \mathrm{a}-\mathrm{c})$ and the stress-displacement formulation in (11.5). For convenience we assume the initial temperature $T_{0}$ to be constant and the reference state. Let $F o=s t / L^{2}$. Then the stress components are given by $\sigma_{x x}=\sigma_{y y}=\sigma$, where

$$
\sigma=-\frac{\alpha E\left(T_{2}-T_{1}\right)}{1-\nu} \sum_{n=0}^{\infty} A_{n}\left[\chi_{n}+6 z^{\prime}\left(J_{n}-2 K_{n}\right)-4 J_{n}+6 K_{n}\right] \exp \left(-F o \lambda_{n}^{2}\right),
$$

with $A_{n}, \chi_{n}$ from $(3.4 \mathrm{a}-\mathrm{c}), \lambda_{n}$ from (3.5), and $J_{n}, K_{n}$ from (3.6). The curvature of the mirror is $R_{x x}=R_{y y}=R$, where

$$
\frac{1}{R} \approx-\frac{2 \alpha\left(T_{2}-T_{1}\right)}{L}\left[c B i_{1}+6 \sum_{n=0}^{\infty} A_{n}\left(-J_{n}+2 K_{n}\right) \exp \left(-F_{o} \lambda_{n}^{2}\right)\right] .
$$




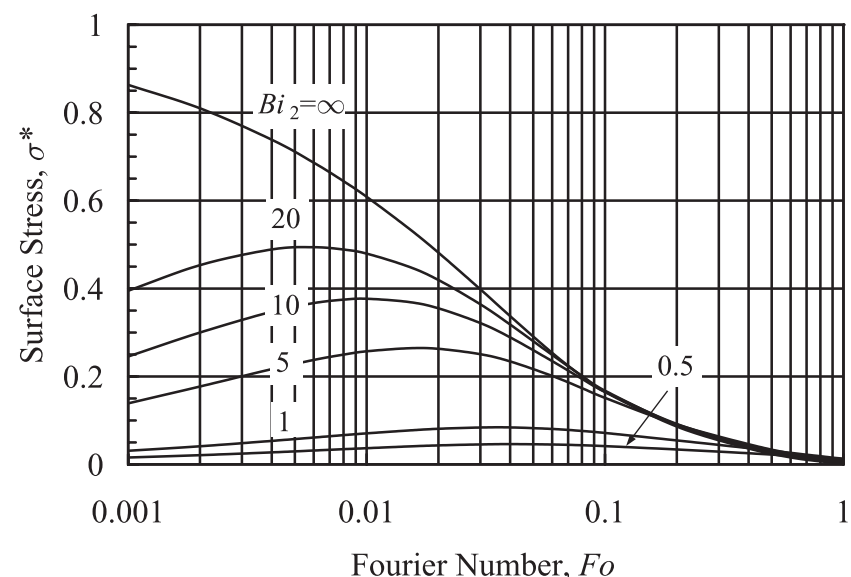

FIG. 5(a). Transient stress response of a free plate with unequal boundary temperatures and unequal heat transfer rates at endpoints. $\sigma_{x x}=\sigma_{y y}=\sigma$, and the ordinate is $\sigma^{*}=-\sigma(1-\nu) /\left[\alpha E\left(T_{2}-T_{1}\right)\right]$. $T_{0}=T_{1}, B i_{1}=1$. Surface stress at $z=L: B i_{2}=0.5,1,5,10,20, \infty$.

In the steady state the stress levels return to zero but the plate retains a curvature that is concave up for $T_{2}<T_{1}$. The steady state curvature is not independent of the plate's thickness; the curvature becomes independent of the thickness when one of the ends is thermally insulated. Transient response of normalized surface stresses, $\sigma^{*}=-\sigma(1-\nu) /\left[\alpha E\left(T_{2}-T_{1}\right)\right]$ at $z=L$, is shown in Fig. 5(a). The maximum stress is at the surface with the larger $\Delta T$ from the boundary temperature, in this case at $z=L$. The maximum stress occurs some time after the thermal shock is introduced. The time lag of occurrence of the max stress increases as $B i_{2}$ decreases. Transient response through the thickness is shown in Fig. 5(b) for $B i_{1}=1, B i_{2}=10$. The stress distribution is unsymmetrical about mid-point because the end conditions are unequal. The results are sensitive to boundary conditions, and in cases like this, the use of an ad hoc simplified boundary conditions can only be approximate. In Fig. 5(c) we show variation with time of the maximum temperature difference $\Delta \theta=\theta(L, t)-\theta(0, t)$, the instantaneous temperature rise $\vartheta^{*}=\left(T(L, t)-T_{0}\right) /\left(T_{2}-T_{1}\right)$, and the surface stress $\sigma^{*}$ at $z=L$. The temperature differences increase monotonically with time until the steady state has been reached. The stress components, on the other hand, have rising and falling characteristics. Evidently, an estimate of the instantaneous quasi-static thermal stress from a consideration of steady state temperatures alone will be conservative.

Mixed boundary conditions. The surface at $z=0$ is held at temperature $T_{1}$ but the surface at $z=L$ is exchanging heat by radiation to space at $T_{2}$. The initial temperature $T_{0}$ is constant. The temperature difference is

$$
\begin{aligned}
\vartheta= & T_{1}-T_{0}+\frac{B i_{2}}{1+B i_{2}}\left(T_{2}-T_{1}\right) z^{\prime} \\
& +\left(T_{2}-T_{1}\right) \sum_{n=0}^{\infty} A_{n} \sin \left(\lambda_{n} z^{\prime}\right) \exp \left(-F_{O} \lambda_{n}^{2}\right) .
\end{aligned}
$$




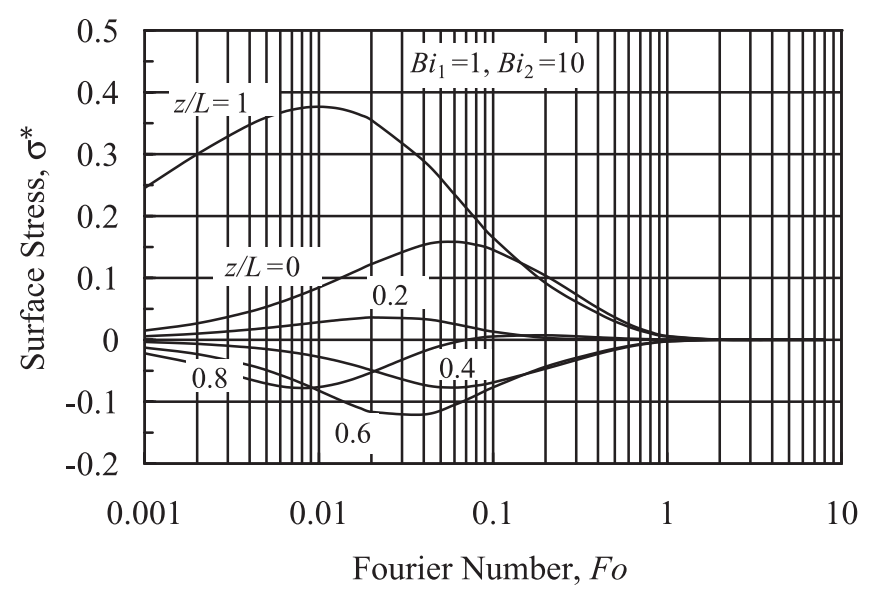

FIG. 5(b). Transient stress response of a free plate with unequal boundary temperatures and unequal heat transfer rates at endpoints. $\sigma_{x x}=\sigma_{y y}=\sigma$, and the ordinate is $\sigma^{*}=-\sigma(1-\nu) /$ $\left[\alpha E\left(T_{2}-T_{1}\right)\right] . \quad T_{0}=T_{1}, B i_{1}=1$. Stress response through the thickness: $B i_{2}=10 . z / L=0,0.2,0.4,0.6,0.8,1$.

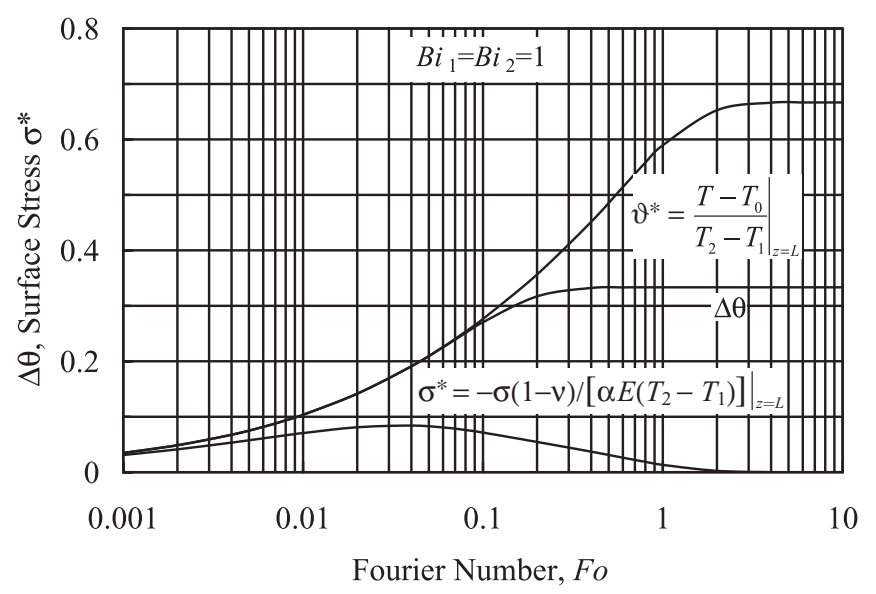

Fig. 5(c). Transient response of surface stress and temperature difference $\Delta \theta$ at $z=L$ of a free plate with unequal boundary temperatures at endpoints. $\theta=\left(T-T_{1}\right) /\left(T_{2}-T_{1}\right), \quad \vartheta^{*}=\left(T-T_{0}\right) /\left(T_{2}-T_{1}\right)$, $\Delta \theta=\theta(L, t)-\theta(0, t)$. The nondimensional stress is $\sigma^{*}=-\sigma(1-\nu) /$ $\left[\alpha E\left(T_{2}-T_{1}\right)\right] . T_{0}=T_{1}, B i_{1}=1, B i_{2}=1$.

The $A_{n}$ are given in (9.1). The eigenvalues are nonnegative roots of $\lambda_{n} \cot \lambda_{n}=-B i_{2}$, $B i_{2} \geq 0$. The stress-displacement for the free plate is given by Eqs. (11.1), (11.5) where

$$
\begin{aligned}
& d_{1}=\frac{\alpha E\left(T_{2}-T_{1}\right)}{(1-\nu) L}\left[\frac{B i_{2}}{1+B i_{2}}+6 \sum_{n=0}^{\infty} A_{n} \frac{1}{\lambda_{n}}\left(-1-\cos \lambda_{n}+\frac{2}{\lambda_{n}} \sin \lambda_{n}\right) \exp \left(-F o \lambda_{n}^{2}\right)\right], \\
& d_{2}=\frac{\alpha E\left(T_{2}-T_{1}\right)}{(1-\nu)}\left[\frac{T_{1}-T_{0}}{T_{2}-T_{1}}+2 \sum_{n=0}^{\infty} A_{n} \frac{1}{\lambda_{n}}\left(2+\cos \lambda_{n}-\frac{3}{\lambda_{n}} \sin \lambda_{n}\right) \exp \left(-F o \lambda_{n}^{2}\right)\right] .
\end{aligned}
$$


Constant temperature end conditions. The ends are maintained at constant temperatures $T_{1}, T_{2}$. Here $B i_{1}=\infty, B i_{2}=\infty$, and the temperature difference is

$$
\vartheta=T_{1}-T_{0}+\left(T_{2}-T_{1}\right) z^{\prime}+\left(T_{2}-T_{1}\right) \sum_{n=1}^{\infty} A_{n} \sin \left(n \pi z^{\prime}\right) \exp \left(-F o n^{2} \pi^{2}\right) .
$$

The Fourier coefficients $A_{n}$ are given in (9.2). For $d_{1}, d_{2}$, set $B i_{2} /\left(1+B i_{2}\right)=1$ and $\lambda_{n}=n \pi$ in $(12.2 \mathrm{~b}-\mathrm{c})$, and then sum from $n=1$ to $\infty$.

Radiation combined with time-varying heat flux at the boundary. The ends are exchanging heat by radiation at unequal heat transfer rates to medium at unequal temperatures. The heat flux $q_{2}(t)$ at $z=L$ is also specified. The initial temperature $T_{0}$ is constant. From (9.8) the solution for $t<\tau_{1}$ is

$$
\begin{aligned}
\vartheta= & T_{1}-T_{0}+c\left(1+B i_{1} z^{\prime}\right)\left(T_{2}-T_{1}\right)+\left(T_{2}-T_{1}\right) \sum_{n=0}^{\infty} A_{n} \chi_{n} \exp \left(-F o \lambda_{n}^{2}\right) \\
& +\frac{c \gamma L t}{\kappa B i_{2}}\left(1+B i_{1} z^{\prime}\right)+\frac{c \gamma L^{3}}{\kappa s B i_{2}} \sum_{n=0}^{\infty} A_{n}^{\prime} \frac{\chi_{n}}{\lambda_{n}^{2}}\left[1-\exp \left(-F o \lambda_{n}^{2}\right)\right], \\
d_{1}= & \frac{\alpha E\left(T_{2}-T_{1}\right)}{(1-\nu) L}\left\{c B i_{1}\left[1+\frac{\gamma L t}{\kappa B i_{2}\left(T_{2}-T_{1}\right)}\right]\right. \\
& +6 \sum_{n=0}^{\infty} A_{n}\left(-J_{n}+2 K_{n}\right) \exp \left(-F o \lambda_{n}^{2}\right) \\
& \left.+\frac{6 c \gamma L^{3}}{\kappa s B i_{2}\left(T_{2}-T_{1}\right)} \sum_{n=0}^{\infty} A_{n}^{\prime} \frac{1}{\lambda_{n}^{2}}\left(-J_{n}+2 K_{n}\right)\left[1-\exp \left(-F o \lambda_{n}^{2}\right)\right]\right\}, \\
d_{2}= & \frac{\alpha E\left(T_{2}-T_{1}\right)}{(1-\nu)}\left\{\frac{T_{1}-T_{0}}{T_{2}-T_{1}}+c\left[1+\frac{\gamma L t}{\kappa B i_{2}\left(T_{2}-T_{1}\right)}\right]\right. \\
& +2 \sum_{n=0}^{\infty} A_{n}\left(2 J_{n}-3 K_{n}\right) \exp \left(-F o \lambda_{n}^{2}\right) \\
& \left.+\frac{2 c \gamma L^{3}}{\kappa s B i_{2}\left(T_{2}-T_{1}\right)} \sum_{n=0}^{\infty} A_{n}^{\prime} \frac{1}{\lambda_{n}^{2}}\left(2 J_{n}-3 K_{n}\right)\left[1-\exp \left(-F o \lambda_{n}^{2}\right)\right]\right\} .
\end{aligned}
$$

If $T_{1}=T_{2}$, then the results will be slightly modified. See (8.2), (8.4) if one or both Biot numbers are zero. The formulation is quite general. Two nondimensional parameters have appeared: $\left(T_{1}-T_{0}\right) /\left(T_{2}-T_{1}\right)$ representative of the boundary temperature specification, and $\gamma L^{3} /\left[\kappa s\left(T_{2}-T_{1}\right)\right]$ representative of the dynamic heating rate at the surface. The Fourier coefficients $A_{n}, A_{n}^{\prime}$ are given in (9.5), (9.6). The curvature due to $q_{2}(t)$ alone is

$$
\frac{1}{R} \approx-\frac{2 \gamma \alpha t}{\kappa} \cdot \frac{c B i_{1}}{B i_{2}}-\frac{12 \gamma \alpha L^{2}}{\kappa s} \cdot \frac{c}{B i_{2}} \sum_{n=0}^{\infty} A_{n}^{\prime} \frac{\left(-J_{n}+2 K_{n}\right)}{\lambda_{n}^{2}}\left[1-\exp \left(-F o \lambda_{n}^{2}\right)\right], \quad t<\tau_{1} .
$$




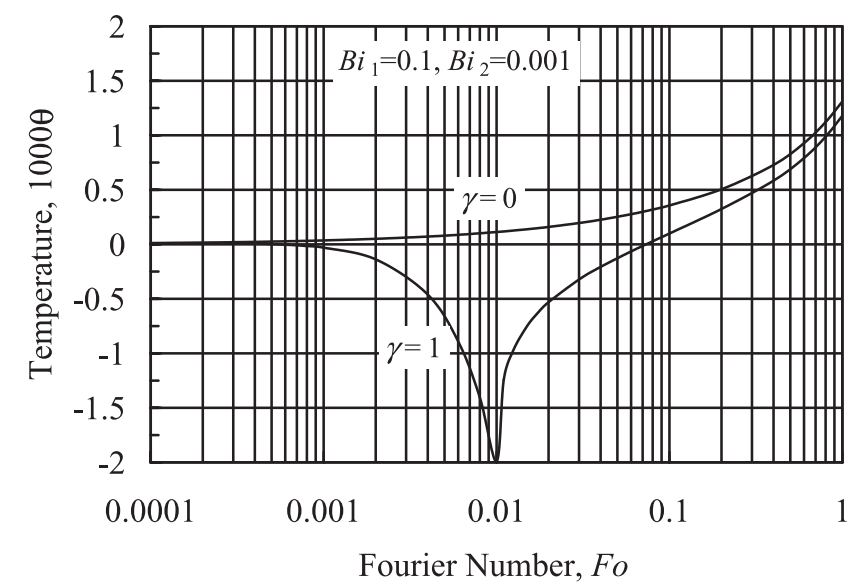

FIG. 6(a). Transient response of a free plate with radiation combined with a time-varying heat influx at the surface. $T_{0}=T_{1}, q_{2}(t)=\gamma t$, $z=L, B i_{1}=0.1, B i_{2}=0.001, \tau_{1}=129 \mathrm{~s}(F o=0.01) T_{1}=293^{\circ} \mathrm{K}$, $T_{2}=5^{\circ} \mathrm{K}, \gamma=1 \mathrm{~W} /\left(\mathrm{m}^{2} \mathrm{~s}\right), \gamma L^{3} /\left[\kappa s\left(T_{2}-T_{1}\right)\right]=-2.8 . \quad \theta=(T-$ $\left.T_{1}\right) /\left(T_{2}-T_{1}\right)$.

The curvature is a function of the plate's thickness, its material properties, and the Biot numbers. Since $B i \propto \epsilon$, the mirror's optical coatings should be selected carefully to keep elastic deformation to the minimum. We omit results for $t>\tau_{1}$.

As an example we assume $\gamma=1 \mathrm{~W} /\left(\mathrm{m}^{2} \mathrm{~s}\right), \tau_{1}=129 \mathrm{~s}\left(F_{O}=0.01\right), L=10 \mathrm{~cm}$, $T_{1}=293^{\circ} \mathrm{K}, T_{2}=5^{\circ} \mathrm{K}$, and $T_{0}=T_{1}$. From Table $2, \gamma L^{3} /\left[\kappa s\left(T_{2}-T_{1}\right)\right]=-2.8$. The temperature and surface stresses for a time-varying flux $q_{2}(t)=\gamma t$ are shown in Figs. 6(a), 6(b). The response is initially slow up to $F o=0.001$. Then the temperature and the surface stresses begin to increase rapidly with time, with $\theta$ becoming more negative because the incoming heat flux is exceeding the heat loss by radiation. The max stress is at $t=\tau_{1}$ when the mirror comes to a full stop, and the heat flux drops to zero. The changes in the temperature and stresses may appear small compared to the zero flux case, but for sensitive astronomical missions a deci- or even a centi-Kelvin change in the mirror's temperature during scientific observation may have to be controlled. The response of the mirror indicates that to minimize the thermal stress and thermal distortion it might be advantageous to complete the mirror's movement in multi steps, rather than in one sweep. Due to the low thermal diffusivity of the mirror material the disturbance is largely concentrated within a thin layer of the surface exposed to the heat flux. Plots of $\theta, \sigma^{*}$ at various $z / L$ (not shown) confirm this. We mention in passing that the formulation is easily adapted to other practical problems such as the cooling of glass in the manufacturing processes, the transpirated cooling of blade tips, or the aerodynamic heating of re-entry bodies.

Comparison with known solutions. We selected two nontrivial cases.

(a) Boley and Weiner [6], p. 283, Carslaw and Jaeger [8, p. 112. The end at $z=0$ is insulated while the end at $z=L$ is exposed to a uniform heat flux which varies with time. 


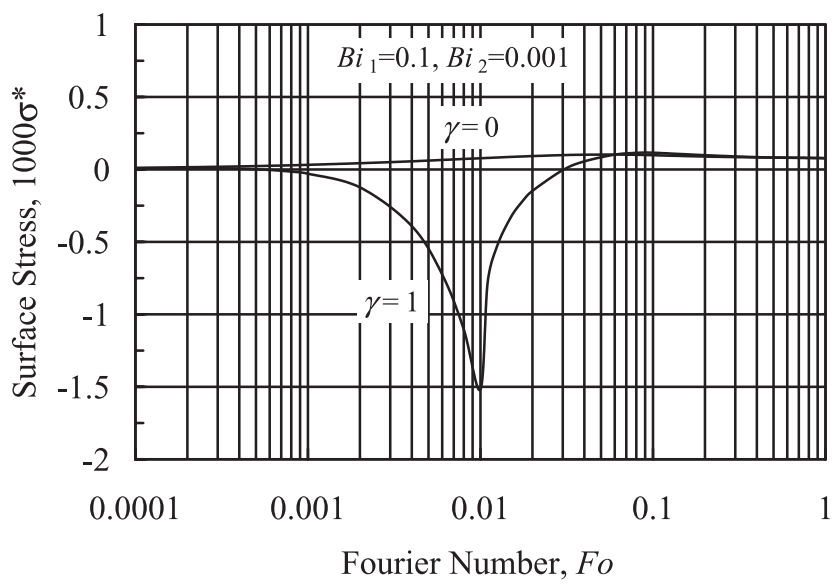

FIG. 6(b). Transient response of a free plate with radiation combined with a time-varying heat influx at the surface. $T_{0}=T_{1}, q_{2}(t)=\gamma t$, $z=L, B i_{1}=0.1, B i_{2}=0.001, \tau_{1}=129 \mathrm{~s}(F o=0.01), T_{1}=$ $293^{\circ} \mathrm{K}, T_{2}=5^{\circ} \mathrm{K}, \gamma=1 \mathrm{~W} /\left(\mathrm{m}^{2} \mathrm{~s}\right), \gamma L^{3} /\left[\kappa s\left(T_{2}-T_{1}\right)\right]=-2.8$. Nondimensional surface stress, $\left.\sigma^{*}=-\sigma(1-\nu) / \alpha \alpha E\left(T_{2}-T_{1}\right)\right]$.

The initial temperature is zero and the initial heat flux is $q_{0}$. This is a special case of the general solution discussed earlier in (8.4). Here $B i_{1}=B i_{2}=0$. The eigenfunctions are $\cos \left(n \pi z^{\prime}\right), n=0,1,2, \ldots$. The solution with $\kappa \partial T / \partial z=q_{0}$ at $z=L$, and the other boundary conditions kept homogeneous is

$$
\begin{aligned}
\Phi_{T}= & \frac{q_{0} L}{\kappa}\left[\frac{s t}{L^{2}}-\frac{1}{6}+\frac{1}{2}\left(z^{\prime}\right)^{2}-2 \sum_{n=1}^{\infty}(-1)^{n} \frac{\cos \left(n \pi z^{\prime}\right)}{n^{2} \pi^{2}} \exp \left(-F o n^{2} \pi^{2}\right)\right] \\
\Phi_{\sigma}= & \frac{\alpha E}{1-\nu} \cdot \frac{q_{0} L}{\kappa}\left[-\frac{1}{12}+\frac{1}{2} z^{\prime}-\frac{1}{2}\left(z^{\prime}\right)^{2}+2 \sum_{n=1}^{\infty}(-1)^{n} \frac{\cos \left(n \pi z^{\prime}\right)}{n^{2} \pi^{2}} \exp \left(-F o n^{2} \pi^{2}\right)\right. \\
& \left.-24\left(2 z^{\prime}-1\right) \sum_{n=1,3,5, \ldots}^{\infty} \frac{1}{n^{4} \pi^{4}} \exp \left(-F o n^{2} \pi^{2}\right)\right]
\end{aligned}
$$

where $\Phi_{\sigma}=\Phi_{\sigma_{x x}}=\Phi_{\sigma_{y y}}$. In this particular case the plate's curvature is independent of its thickness. Temperature solution for the time-varying heat flux $q_{2}(t)$ at $z=L$ is

$$
T\left(z^{\prime}, t\right)=\frac{q_{2}(0)}{q_{0}} \Phi_{T}\left(z^{\prime}, t\right)+\int_{0}^{t} \frac{1}{q_{0}} \frac{d q_{2}(\tau)}{d \tau} \Phi_{T}\left(z^{\prime}, t-\tau\right) d \tau .
$$

The stress due to the time-varying heat flux is obtained by substituting $\Phi_{\sigma}$ for $\Phi_{T}$ in (12.7). Equations (12.5), (12.6) fully agree with Boley and Weiner after a simple coordinate transformation.

(b) Hlinka et al. 16, Heisler [14. The ends are suddenly exposed to medium at $T_{\infty}$ through the convective heat transfer coefficient $h$. The initial temperature is zero. This is a particular case of (3.8). Here $B i_{1}=B i_{2}=B i, T_{0}=0$, and $T_{1}=T_{2}=T_{\infty}$. The 




FIG. 7(a). Transient response of a free plate exchanging heat by radiation to medium at temperature $T_{\infty}$. Initial temperature $T_{0}=0$, $T_{1}=T_{2}=T_{\infty}, z=L . B i_{1}=B i_{2}=B i$. Nondimensional surface temperature, $T / T_{\infty}: B i=0.1,1,2,5,10,20, \infty$.

solution is

$$
\left.\begin{array}{rl}
\frac{T}{T_{\infty}}= & -\sum_{n=0}^{\infty} \frac{2 B i^{2}}{\lambda_{n}^{2}+B i^{2}+2 B i}\left(1-\cos \lambda_{n}+\frac{\lambda_{n}}{B i} \sin \lambda_{n}\right) \frac{\chi_{n}}{\lambda_{n}} \exp \left(-F o \lambda_{n}^{2}\right), \\
\sigma_{x x}= & \sigma_{y y}=\frac{\alpha E T_{\infty}}{1-\nu}\left\{\sum_{n=0}^{\infty} \frac{2 B i^{2}}{\lambda_{n}^{2}+B i^{2}+2 B i}\left(1-\cos \lambda_{n}+\frac{\lambda_{n}}{B i} \sin \lambda_{n}\right)\right. \\
& \left.\times \frac{1}{\lambda_{n}}\left[\chi_{n}+6 z^{\prime}\left(J_{n}-2 K_{n}\right)-4 J_{n}+6 K_{n}\right] \exp \left(-F o \lambda_{n}^{2}\right)\right\} .
\end{array}\right\}
$$

The eigenfunction is $\chi_{n}=\sin \left(\lambda_{n} z^{\prime}\right)+\left(\lambda_{n} / B i\right) \cos \left(\lambda_{n} z^{\prime}\right)$. The $\lambda_{n}$ are nonnegative roots of $\beta_{n} \tan \beta_{n}=C$ and $\beta_{n} \cot \beta_{n}=-C$ where $\beta_{n}=\frac{1}{2} \lambda_{n}, C=\frac{1}{2} B i$. The integrals $J_{n}, K_{n}$ were given in (3.6). The problem is symmetrical about the centerline at $z=\frac{1}{2} L$. To show that the solution is symmetric we note that

$$
1-\cos \lambda_{n}+\frac{\lambda_{n}}{B i} \sin \lambda_{n}=1-\cos 2 \beta_{n}+\frac{2 \beta_{n}}{B i} \sin 2 \beta_{n}=2 \sin ^{2} \beta_{n}\left(1+\frac{2 \beta_{n}}{B i} \cot \beta_{n}\right) .
$$

In view of the second eigenvalue equation the term vanishes identically for the odd $n$, leaving the solution symmetric in $z$. The transient response curves have an upper envelope defined by $B i=\infty$ (constant temperature end condition),

$$
\left.\begin{array}{l}
\frac{T}{T_{\infty}}=1-4 \sum_{n=1,3,5, \ldots}^{\infty} \frac{\sin \left(n \pi z^{\prime}\right)}{n \pi} \exp \left(-F_{o} n^{2} \pi^{2}\right), \\
\sigma_{x x}=\sigma_{y y}=\frac{4 \alpha E T_{\infty}}{1-\nu} \sum_{n=1,3,5, \ldots}^{\infty} \frac{1}{n \pi}\left[\sin \left(n \pi z^{\prime}\right)-\frac{2}{n \pi}\right] \exp \left(-F o \lambda_{n}^{2} \pi^{2}\right) .
\end{array}\right\}
$$






Fourier number, $F_{O}$

FIG. 7(b). Transient response of a free plate exchanging heat by radiation to medium at temperature $T_{\infty}$. Initial temperature $T_{0}=0$. $T_{1}=T_{2}=T_{\infty}, z=L . B i_{1}=B i_{2}=B i$. Nondimensional surface stress, $\sigma^{*}=-\sigma(1-\nu) /\left(\alpha E T_{\infty}\right): B i=1,2,5,10,20, \infty$.

The maximum stress occurs at the surface for $B i=\infty$ at $t=0$ when the thermal shock is introduced, and its magnitude is

$$
\left.\sigma_{x x}\right|_{\max }=\left.\sigma_{y y}\right|_{\max }=-\frac{4 \alpha E T_{\infty}}{1-\nu} \cdot \frac{2}{\pi^{2}} \sum_{n=1,3,5, \ldots}^{\infty} \frac{1}{n^{2}}=-\frac{\alpha E T_{\infty}}{1-\nu} .
$$

The stress is compressive for $T_{\infty}-T_{0}>0$ and tensile for $T_{\infty}-T_{0}<0$. For finite values of $B i$ the time of occurrence of the max stress increases as the Biot number decreases. Plots of $T / T_{\infty}$ and $-\sigma(1-\nu) /\left(\alpha E T_{\infty}\right)=\sigma / \sigma_{\max }$ are shown in Figs. $7(\mathrm{a}), 7(\mathrm{~b})$. The results are self-explanatory. They agree with Hlinka et al. and Heisler's numerical results presented in graphical charts and correlations.

Acknowledgment. The author wishes to acknowledge his indebtedness to Professor I. M. Cohen who introduced him to asymptotic theory, and to Professor S. C. Batterman who taught elasticity and plasticity.

Appendix A. Roots of $\lambda \tan \lambda=C, \lambda \cot \lambda=-C$.

Because of their frequent occurrences the transcendental equations

$$
\lambda \tan \lambda=C, \quad C>0,
$$

and

$$
\lambda \cot \lambda=-C, \quad C>-1,
$$

where $C$ is some constant (cf. $\S 6$ ) are of interest. The restrictions on $C$ are to insure the roots are real. The restriction is necessary because eigenvalues of a regular SturmLiouville system are real. The negative values of $C$ in (A.2) occur in spherical geometries. 
Fourier's original investigation was on the cooling of spherical bodies for which he investigated roots of the equation $n X / \tan n X=1-h X$, where $X$ is radius, $h$ the heat transfer coefficient, and $n$ any positive number. Let $\varepsilon=n X$ and $\beta=1-h X$, so that $\varepsilon / \beta=\tan \varepsilon$. Fourier observes (Théorie Analytique de la Chaleur, 1822) that when $\varepsilon / \beta$ is small the root will be approximated as

$$
\varepsilon=\cdots \tan ^{-1}\left\{\frac{1}{\beta} \tan ^{-1}\left[\frac{1}{\beta} \tan ^{-1}\left(\frac{1}{\beta} \tan ^{-1} \frac{1}{\beta}\right)\right]\right\} .
$$

In this, Fourier seems to have anticipated asymptotic determination of the roots given here. However, further improvement by asymptotic iteration cannot be recommended; see de Bruijn [11. Solutions to (A.1)-A.2) are summarized below for completeness (cf. [1], 8], 18 for partial tabulation of the roots), although the general solutions given in $\S \S 5$ and 6 may also be used.

Roots of $\lambda \tan \lambda=C$.

(a) $\lambda<C$

$$
\lambda_{n}=p^{\prime} C+\frac{1}{3}\left(1-\frac{1}{C}+\frac{1}{C^{2}}\right) p^{\prime 3}-\frac{1}{5}\left(1-\frac{8}{3 C}+\frac{10}{3 C^{2}}\right) p^{\prime 5}+O\left(p^{\prime 7}\right),
$$

in which $p^{\prime}=\left(n+\frac{1}{2}\right) \pi /(C+1), n=0,1,2, \ldots$

(b) $\lambda>C$

$$
\begin{aligned}
\lambda_{0}= & \sqrt{C}\left(1-\frac{1}{3} C+\frac{8}{15} C^{2}-\frac{1}{3} C^{3}\right)+O\left(C^{\frac{9}{2}}\right), \\
\lambda_{n}= & p+\frac{C}{p}-C^{2}\left(1+\frac{1}{3} C\right) \frac{1}{p^{3}} \\
& +C^{3}\left(2+\frac{4}{3} C+\frac{1}{5} C^{2}\right) \frac{1}{p^{5}}+O\left(\frac{1}{p^{7}}\right) \quad(n>0) .
\end{aligned}
$$

Here $p=n \pi, n=1,2,3, \ldots$. Let $M=\operatorname{round}[C / \pi]$, where round $[x]$ is the integer nearest to $x$. Then use (A.3) for $n<M$ and (A.4) for $n \geq M$. For example, if $C=5$, use (A.3) for $n<2$ and (A.4) for $n \geq 2$.

Roots of $\lambda \cot \lambda=-C$. Note that $\lambda=0$ is not an admissible root when $C=0$.

(a) $\lambda<C$

$$
\lambda_{n}=C q^{\prime}+\frac{1}{3}\left(1-\frac{1}{C}+\frac{1}{C^{2}}\right) q^{\prime 3}-\frac{1}{5}\left(1-\frac{8}{3 C}+\frac{10}{3 C^{2}}\right) q^{\prime 5}+O\left(q^{\prime 7}\right),
$$

where $q^{\prime}=(n+1) \pi /(C+1), n=0,1,2, \ldots$.

(b) $\lambda>C$

$$
\lambda_{n}=q+\frac{C}{q}-C^{2}\left(1+\frac{1}{3} C\right) \frac{1}{q^{3}}+C^{3}\left(2+\frac{4}{3} C+\frac{1}{5} C^{2}\right) \frac{1}{q^{5}}+O\left(\frac{1}{q^{7}}\right),
$$

where $q=\left(n+\frac{1}{2}\right) \pi, n=0,1,2, \ldots$. Let $N=\operatorname{round}\left[C / \pi-\frac{1}{2}\right]$. Then use A.5 for $n<N$ and (A.6) for $n \geq N$. For example, if $C=5$, use A.5 for $n=0$ and A.6) for $n \geq 1$. If $C=0$, use (A.6) for $n \geq 0$. 


\section{REFERENCES}

[1] M. Abramowitz and I. A. Stegun, Handbook of Mathematical Functions, Dover reprint, New York, 1970. See tables 4-19, 4-20 for the eigenvalues of (A.1), (A.2).

[2] R. C. F. Bartels and R. V. Churchill, Resolution of Boundary Problems by the use of a Generalized Convolution, Bull. Amer. Math. Soc. 48, 276-282 (1942). MR0005994(3:243g)

[3] M. A. Biot, Thermoelasticity and Irreversible Thermodynamics, J. Appl. Phys. 27, 240-253 (1956). MR0077441 (17:1035e)

[4] G. Birkhoff and G-C. Rota, Ordinary Differential Equations, 4th ed, John Wiley, New York, 1989. MR0972977 (90h:34001)

[5] L. M. K. Boelter, V. H. Cherry, H. A. Johnson, R. C. Martinelli, Heat Transfer Notes, McGraw-Hill, New York, 1965.

[6] B. A. Boley and J. H. Weiner, Theory of Thermal Stresses, Mineola (Dover reprint), New York, 1997.

[7] D. E. Carlson, Linear Thermoelasticity, in Handbuch der Physik, Bd. VIa/2, Springer-Verlag, Berlin, 1972.

[8] H. S. Carslaw and J. C. Jaeger, Conduction of Heat in Solids, 2nd ed, Clarendon Press, Oxford, 1973; Reprint of 2nd ed., 1988. MR0959730 (89f:80004)

[9] W. A. Day, Justification of the Uncoupled and Quasi-Static Approximations in a Problem of Dynamic Thermoelasticity, Arch. Rational Mech. Anal. 77, 387-396 (1981). MR.0642554 (83b:73091)

[10] W. A. Day, The Status of the Heat Equation, in Rational Thermodynamics, 2nd ed, (Ed. C. Truesdell), Springer-Verlag, New York, 1984. Also, Heat Conduction within Linear Thermoelasticity, Springer Tracts in Natural Philosophy, vol. 30, Springer-Verlag, Berlin, 1985. MR0804043 (87c:73001)

[11] N. G. de Bruijn, Asymptotic Methods in Analysis, 2nd ed, North-Holland, Amsterdam, 1961. MR 0177247 (31:1510)

[12] G. H. Hardy, Collected Papers, vol. III, Clarendon Press, Oxford, 1969. MR0255362 (41:24)

[13] G. H. Hardy and W. W. Rogosinski, Fourier Series, Cambridge Tracts No. 38, 3rd ed, Cambridge University, Cambridge, 1950. MR0044660 (13:457b)

[14] M. P. Heisler, Temperature Charts for Induction and Constant-Temperature Heating, ASME Trans. 69, 227-236 (1947).

[15] M. P. Heisler, Transient Thermal Stresses in Slabs and Circular Pressure Vessel, J. Appl. Mech. 20, 261-269 (1953).

[16] J. H. Hlinka, H. G. Landau, and V. Paschkis, Charts on Elastic Thermal Stresses in Heating and Cooling of Slabs and Cylinders, ASME paper 57-A-238, 1-19 (1957).

[17] E. Landau, Foundations of Analysis, 3rd ed, (English translation by F. Steinhardt), Chelsea/AMS reprint, 2000. MR0038404 (12:397m)

[18] A. V. Luikov, Analytical Heat Diffusion Theory, Academic Press, London, 1968.

[19] N. Noda, R. B. Hetnarski, and Y. Tanigawa, Thermal Stresses, 2nd ed, Taylor and Francis, New York, 2003.

[20] F. W. J. Olver, Asymptotics and Special Functions, Academic Press, New York, 1974; Reprint AK Peters, Ltd., Wellesley, MA, 1997. MR0435697(55:8655) MR.1429619 (97i:41001)

[21] M. N. Özişik, Heat Conduction, 2nd ed, Wiley-Interscience, New York, 1993.

[22] P. J. Schneider, Temperature Response Charts, John Wiley, New York, 1963. [A total of 120 uniformly sized charts (mostly $10.5 \mathrm{~cm} \times 21 \mathrm{~cm}$ ) were specially prepared for this book.]

[23] S. P. Timoshenko and J. N. Goodier, Theory of Elasticity, 3rd ed, McGraw-Hill, New York, 1970.

[24] E. C. Titchmarsh, The Theory of Functions, 2nd ed, Clarendon Press, Oxford, 1985.

[25] G. P. Tolstov, Fourier Series (English translation by Richard. A. Silverman), Dover reprint, New York, 1962. MR0425474 (54:13429)

[26] J. H. Weiner, A Uniqueness Theorem for the Coupled Thermoelastic Problem, Quart. Appl. Math. 15, 102-105 (1957). MR0088216 (19:484i)

[27] R. D. S. Zerkle and J. E. Sunderland, The transient temperature distribution in a slab subject to thermal radiation, J. Heat Transfer, Trans. ASME. 87, 117-133 (1965).

[28] A. Zygmund, Trigonometric Series, 2nd ed, Cambridge University Press, Cambridge, 1988. MR0933759 (89c:42001) 